\title{
Schiaparelli et les archéologues italiens aux bords du Nil : égyptologie et rivalités diplomatiques entre 1882 et 1922
}

\author{
Carole Jarsaillon
}

In 1903, Ernesto Schiaparelli made the decision to excavate directly in Egypt rather than purchasing objects for the Museo Egizio of Turin on the art market. The MAI, the Italian Archaeological Mission in Egypt, was thus born, marking the first steps of the newly unified Italian nation-state in the field of Egyptian archaeology. When investigating the context of these early Italian excavations and the way the thriving science of Egyptology was managed in Egypt between 1882 and 1922, one notices the paramount importance of geopolitical stakes in the matter, and particularly the prominence of Anglo-French rivalry. The article explores how Italian Egyptology managed to thrive in this international political context, and seeks to assess to what extent these diplomatic stakes impacted the work of Schiaparelli's team and its dissemination among the international Egyptological community. The author first looks into the organization of Egyptology and its Service of Antiquities as a tool of colonialism, as well as a political stake among European powers at the turn of the century, especially as regards the internationalization and expansion of archaeological excavations along the banks of the Nile. She then turns to the place of Italian Egyptology within this framework, and notably its relationship with the Service of Antiquities, its discoveries, and how knowledge about these discoveries was disseminated through official publications.

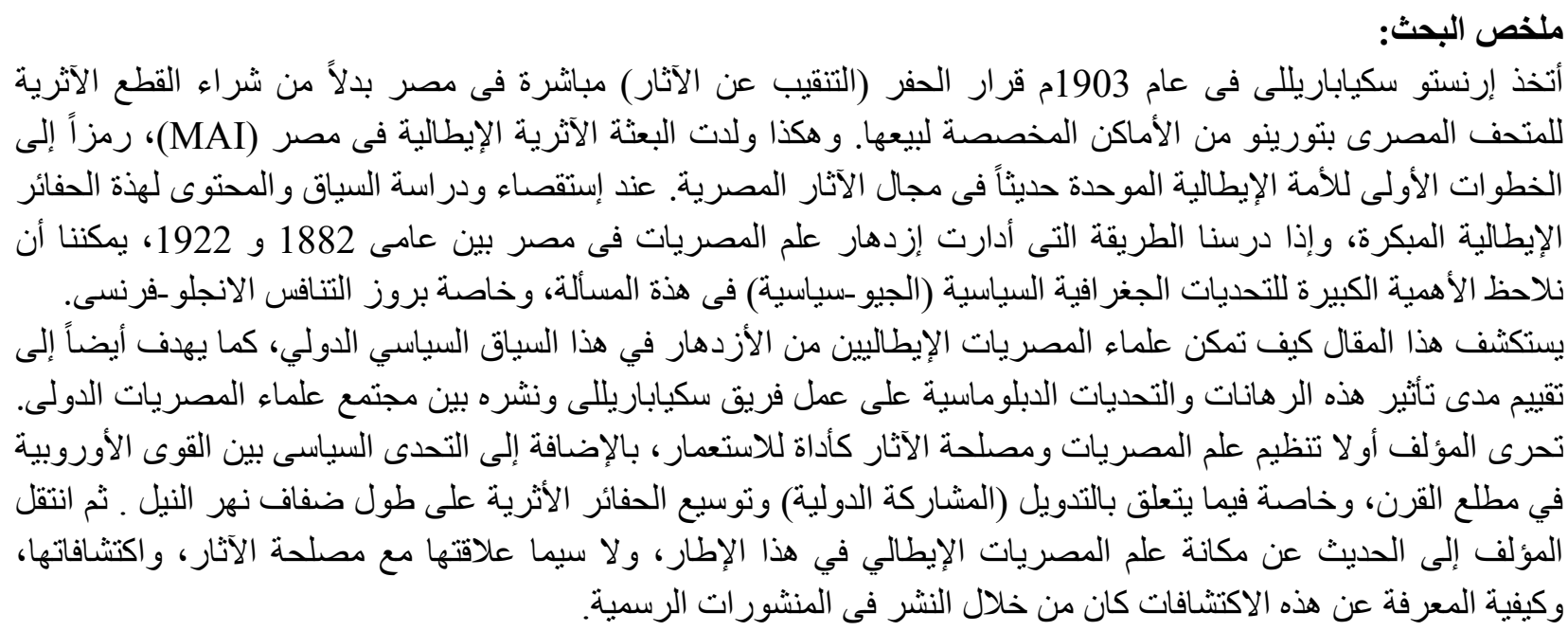

Une suite de noms et de découvertes : c'est ainsi qu'Andrew Bednarski ${ }^{1}$ évoque ce à quoi s'est longtemps réduite l'histoire de l'égyptologie. Cette histoire s'avère pourtant bien plus riche qu'une liste de trésors et d'explorateurs, mais surtout plus pertinente pour les acteurs de l'égyptologie actuelle. Comme le montre l'exposition Missione Egitto 19031920, présentée par le Museo Egizio de Turin en 2017, ${ }^{2}$ les conservateurs de musées sont les premiers à tenter de comprendre l'histoire des objets qu'ils exposent, ${ }^{3}$ ou plus exactement, leurs histoires, car après leur vie antique, ces artefacts connaissent, une fois découverts, un second parcours tout aussi fascinant à l'époque moderne. En partant à la recherche de celui-ci, c'est aussi l'aventure des hommes qui les ont mis au jour, le contexte de leur découverte, en somme l'histoire de l'égyptologie elle-même qui se dévoilent. Cette histoire a souvent été étudiée pour elle-même, pourtant, elle est indissociable de l'histoire générale, méditerranéenne, 
européenne, et mondiale : ce n'est qu'à la lumière du contexte géopolitique plus vaste au cœur duquel évoluent les acteurs de l'égyptologie que peuvent se comprendre les motifs et les modalités selon lesquels cette science s'est développée. Le contexte colonial européen tient bien sûr un rôle de premier plan s'agissant de l'Egypte au tournant du XIX ${ }^{\text {ème }}$ et du XX ${ }^{\text {ème }}$ siècle. Si l'Italie suit l'exemple de ses voisins européens dans la ruée vers l'Afrique, ${ }^{4}$ son rôle en Egypte peut paraître secondaire par rapport à celui du Royaume-Uni ou de la France. Pourtant, elle y est bien présente, ${ }^{5}$ et les relations entre ces différentes puissances, sur les bords du Nil et plus largement sur le continent africain, se révèlent avoir un impact plus important sur l'histoire de l'égyptologie italienne qu'on ne pourrait le penser.

En effet, Ernesto Schiaparelli en 1903, en faisant le choix des fouilles plutôt que du marché de l'art pour compléter la collection du Museo Egizio de Turin, entraîne la création de la Mission Archéologique Italienne en Egypte, marquant ainsi les premiers pas de l'Italie sur le terrain de fouilles égyptien. ${ }^{6}$ L'entrée italienne dans le jeu archéologique égyptien se fait donc au cœur d'une période d'effervescence de l'égyptologie, qui connaît un âge d'or entre 1882 et 1922. Celui-ci s'explique en partie par l'importante expansion des fouilles, tant en nombre qu'en moyens mobilisés, grâce à l'internationalisation du Service des Antiquités qui était depuis sa création par Mariette en 1858, resté franco-égyptien. Quel est alors le contexte précis dans lequel se déroulent les premières fouilles italiennes dans la vallée du Nil ? Comment l'égyptologie est-elle administrativement gérée, par qui, et selon quelles modalités ? C'est en se penchant sur ces questions que les enjeux géopolitiques, et en particulier la rivalité franco-britannique, apparaissent comme des acteurs de premier plan dans le développement de l'archéologie égyptienne de l'époque. Cet article s'attache ainsi à montrer comment l'égyptologie italienne a su tirer parti du contexte politique international pour faire ses preuves sur le terrain archéologique égyptien. Il tente de définir dans quelle mesure ces enjeux diplomatiques ont un impact sur les découvertes faites par Schiaparelli et ses confrères, sur leur déroulement et sur leur postérité.

\section{Politique et administration de l'Egypte au début des années 1880 : l'instrumentalisation de l'égyptologie par les puissances coloniales}

Ernesto Schiaparelli s'inscrit dans ce que l'on qualifie souvent d’âge d'or de l'égyptologie européenne : la quarantaine d'années qui s'étend entre le début des années 1880 - marquant l'arrivée de Gaston Maspero, successeur d'Auguste Mariette à la tête du Service des Antiquités Egyptiennes - et 1922, marquée par la découverte d'Howard Carter dans la Vallée des Rois. Outre ces évènements scientifiques, la période coïncide avec un autre élément majeur de l'histoire de l'Egypte moderne : de 1882 à 1922, le pays est placé sous protectorat britannique, suite à un long processus d'ingérence de la part du Royaume-Uni et de la France. En effet, la politique de modernisation du pays entreprise par Ismaill Pacha dans les années 1860 mène l'Egypte à un endettement qui la place progressivement sous la tutelle de ses créanciers européens : la France, qui possède la moitié des parts du Canal de Suez, inauguré en 1869, et le RoyaumeUni, qui rachète l'autre moitié à une Egypte déjà fortement endettée en 1875. Ceux-ci, sous couvert de la protection de leurs intérêts financiers - le Canal de Suez était d'une importance cruciale pour la route des Indes, et Alexandrie jouait un rôle de premier plan dans le commerce maritime méditerranéen s'accaparent graduellement une place importante au sein du gouvernement égyptien. Si bien qu'Ismail Pacha, peu coopératif aux yeux des Européens, se voit contraint par les pressions conjointes de Paris, Londres et Istanbul, d'abdiquer en faveur de son fils Tawfiq Pacha en 1879. Mais l'ingérence européenne ne contrarie pas seulement le Vice-roi, et bientôt, ce sont les Egyptiens qui se révoltent contre le condominium franco-britannique : le mouvement nationaliste mené par Arabi Pacha en 1881 plonge l'Egypte dans une instabilité qui aboutit au protectorat de 1882, lorsqu'une rixe à Alexandrie offre l'occasion aux Britanniques d'envoyer des forces armées. La France quant à elle, divisée entre la Question Allemande et le problème colonial, laisse son voisin d'Outre-Manche gérer la situation $:^{8}$ une décision qui n'est pas sans conséquence, puisque la nouvelle prédominance du Royaume-Uni sur les bords du Nil compromet la position influente des Français sur le 
terrain égyptologique, entretenue depuis le début du XIX ${ }^{\text {ème }}$ siècle.

Ainsi, dès 1882, derrière chaque membre du gouvernement égyptien se cache en réalité une éminence grise britannique, détenant la réalité du pouvoir. De fait, au début du XX⿳亠丷⿵冂丶 ${ }^{2}$ siècle, le Ministère des Travaux Publics, duquel dépendent les organismes gérant l'égyptologie, bien qu'officiellement dirigé par Fakhry Pacha, suit dans la pratique les directives du Britannique Sir William Garstin. ${ }^{9}$ Ce dernier a donc autorité sur les deux corps administratifs gérant les affaires égyptologiques : le Comité d'Archéologie d'une part, qui réunit de hauts-fonctionnaires du gouvernement, siégeant pour valider les grandes décisions sur un plan purement administratif, et le Service des Antiquités Egyptiennes d'autre part, qui organise, administre, et supervise scientifiquement les fouilles ainsi que la protection du patrimoine archéologique du pays. ${ }^{10}$ Créé par Auguste Mariette en 1858, ce Service a le statut particulier d'avoir été mis en place et dirigé par des ressortissants français, ${ }^{11}$ tout en étant mis au service du gouvernement égyptien, dont il fait partie - il dépend directement du Khédive jusqu'en 1878 puis du Ministère des Travaux Publics avant de passer à celui de l'Instruction Publique. ${ }^{12}$ Cela créé une double ambiguité : la première, quant à la paternité du Service, se pose lors de la mort de Mariette en 1881. En effet, si le Service a techniquement été créé par un Français, ça n'était en aucun cas une mission lui ayant été confiée par son gouvernement, et plusieurs indices poussent à penser que l'initiative était plus motivée par des convictions personnelles et professionnelles que par un sentiment national. ${ }^{13}$ Pour autant, dès le départ, Mariette a bien conscience de ce que ce Service initialement composé seulement de lui-même, d'un inspecteur lui aussi français, et de quelques réis égyptiens - apporterait à " l'influence française » en Egypte. ${ }^{14}$ Par ailleurs, on ne peut laisser de côté la haute estime de Saïd Pacha pour la France, qui sans doute a joué un rôle dans l'acceptation de la proposition par le Vice-roi en 1858. La conséquence de tout ceci se retrouve dans une appropriation, à tort ou à raison, de l'œuvre de Mariette par la France, qui considère le Service comme son apanage, tout en étant consciente de son impuissance officielle, et qui s'inquiète ainsi de ce qu'il pourrait en advenir à la mort de l'égyptologue. ${ }^{15}$ Le danger, en 1881 , vient plutôt de l'Allemagne, qui en effet, tente de faire pression sur l'Egypte pour soutenir la candidature d'H. Brugsch. A ce moment-là, les Britanniques, qui commencent déjà à tenir les ficelles du gouvernement égyptien, ont plus d'intérêts à contenter leur allié du condominium que l'Allemagne : cédant aux demandes inquiètes des Français, ils soutiennent ainsi le candidat de ces derniers. Après quelques rebondissements, c'est finalement un élève de Mariette, Gaston Maspero, qui lui succède. ${ }^{16}$ La seconde ambiguïté, quant à savoir de qui le Service des Antiquités devrait défendre les intérêts, apparaît dès l'année suivante, lorsque le Service dirigé par un Français doit rendre des comptes à un Ministère égyptien, désormais aux mains des Britanniques.

Dès lors, la rivalité géopolitique opposant les deux puissances coloniales, qui s'étaient déjà déchirées sur le sol égyptien au début du XIX ${ }^{\text {ème }}$ siècle, se propage au cœur même de l'administration égyptologique : l'Egypte moderne est sous autorité britannique, mais la France, à travers le Service des Antiquités qu'elle considère désormais comme un héritage propre, entend bien garder la main sur l'Egypte ancienne. ${ }^{17} \mathrm{Il}$ faut noter ici que le Service, bien qu'instrumentalisé comme nous le verrons par les deux puissances coloniales, est officiellement supposé représenter les intérêts de l'Egypte, et non ceux de la France ou du Royaume-Uni, quand bien même il est géré par des ressortissants de ces pays. ${ }^{18}$ Cette tension créée par le Service des Antiquités, dirigé par un Français, et pourtant placé sous l'autorité du Ministère, géré par les Britanniques, provoque non seulement des conflits d'autorité de faible répercussion, comme cela a pu arriver sous la direction de Victor Loret à la tête du Service, en $1899,{ }^{19}$ mais fait surtout de l'égyptologie un instrument de pouvoir dans cette lutte géopolitique. Les tensions diplomatiques façonnent ainsi le développement et l'histoire de cette science.

En effet, Maspero, plus diplomate que son prédécesseur, ${ }^{20}$ a souvent été qualifié d'anglophile, et fut, en retour, apprécié des Anglais. Ainsi jusqu'en 1882, il est un appui important pour Amelia Edwards dans la mise en place de l'Egypt Exploration Fund. Mais le changement de rapports de force entraîné par le protectorat britannique laisse entrevoir une certaine méfiance : Maspero consulte le Quai d'Orsay sur le 
sujet, montrant par là qu'il comprend la menace que peut représenter pour l'égyptologie française cette nouvelle configuration politique. ${ }^{21}$ De même, la lecture de sa correspondance privée tend également à nuancer sa présumée anglophilie. ${ }^{22}$ Pour autant, c'est aussi lui qui fait pression sur l'Egypte l'année suivante, pour obtenir l'autorisation exceptionnelle pour l'EEF de fouiller et de rapporter une partie des objets à Londres. Il semble qu'il ait alors simplement en tête l'intérêt du patrimoine égyptien et l'urgence de le mettre au jour et de le conserver, ce pour quoi il considère que toute aide est bonne à prendre. ${ }^{23}$ Ainsi, Maspero semble plus favorable à une coopération européenne en général qu'animé d'une anglophilie particulière. Néanmoins, dans le contexte de la période, l'intérêt diplomatique se trouve dans leur camp : il s'agit également de maintenir la France dans son rôle de premier plan au sein du domaine égyptologique, en se faisant toléré voire apprécié des Britanniques au pouvoir. Ainsi, contrairement à ses prédécesseurs, et à certains de ses successeurs comme Loret, il adopte une tactique de coopération apparente afin de garder la main sur les enjeux plus importants. Elle s'avère payante lorsqu'en 1904, cette tolérance britannique pour la mainmise des Français sur le Service des Antiquités est rendue officielle dans le traité dit de l'Entente Cordiale. Comme le remarque en effet Eric Gady, ${ }^{24}$ il est frappant de constater que cette mention est inscrite dès le tout premier article du contrat passé pour assurer une forme de trêve entre les deux puissances coloniales :

\section{"Article I. Le Gouvernement de Sa Majesté}

Britannique déclare qu'il n'a pas l'intention de changer l'état politique de l'Égypte. De son côté, le Gouvernement de la République Française déclare qu'il n'entravera pas l'action de l'Angleterre dans ce pays en demandant qu'un terme soit fixé à l'occupation Britannique ou de toute autre manière, et qu'il donne son adhésion au projet de Décret Khédivial qui est annexé au présent Arrangement, et qui contient les garanties jugées nécessaires pour la sauvegarde des intérêts des porteurs de la Dette Égyptienne, mais à la condition qu'après sa mise en vigueur aucune modification n'y pourra être introduite sans l'assentiment des Puissances Signataires de la
Convention de Londres de 1885. Il est convenu que la Direction Générale des Antiquités en Égypte continuera d'être, comme par le passé, confiée à un savant Français. Les écoles Françaises continueront à jouir de la même liberté que par le passé . $^{25}$

La place de premier plan prise par l'égyptologie dans cet accord politique est suffisamment remarquable pour frapper les contemporains eux-mêmes, à commencer par Maspero, qui parle «d'une science à laquelle nous attachons assez d'importance pour la faire figurer dans un traité ». ${ }^{26}$ L’utilisation de l'égyptologie comme outil politique au service des enjeux diplomatiques avait pourtant déjà eu lieu par le passé. En 1898, la tension entre les deux puissances était à son comble en Egypte, suite à l'incident de Fachoda. Les Français en sont ressortis diplomatiquement affaiblis, ce qui a sans doute encouragé les Anglais à intervenir dans les affaires du Service des Antiquités en 1899 pour mettre fin à la direction de Loret et soutenir le retour de Maspero pour un second mandat : ${ }^{27}$ un Français qu'ils appréciaient tout particulièrement pour sa collaboration, notamment lors de la création de l'Egypt Exploration Fund comme nous l'avons vu précédemment. En 1902, un évènement vient néanmoins jouer en faveur des Français, expliquant peut-être la bienveillance du traité de 1904 : c'est la signature du traité Prinetti-Barrère, entre la France et l'Italie, par lequel les deux pays s'engagent à rester neutres et à respecter la liberté d'action de l'autre dans la zone méditerranéenne s'étendant de la Cyrénaïque au Maroc. Dès lors, l'Italie devient une sorte d'alliée de la France en Méditerranée. Son arrivée sur le terrain égyptologique en 1903 n'est donc pas sans conséquence sur les rivalités franco-anglaises en Egypte : elle renforce la position française, et a pu jouer en faveur de la France en 1904, poussant les Anglais à accepter de pérenniser l'apanage français de la direction du Service. ${ }^{28}$ Les traités et évènements diplomatiques structurant les relations entre puissances coloniales, qu'il s'agisse de la France, de l'Angleterre ou de l'Italie, rythment ainsi l'administration et les fouilles égyptologiques. L'Allemagne est bien sûr le quatrième protagoniste dans ces rapports de forces européens autour de l'égyptologie, nous aurons l'occasion d'y revenir. Elle pré- 
cède d'ailleurs chronologiquement la rivalité britannique : après la guerre franco-prussienne de 1870, la question allemande occupe le cœur des relations diplomatiques du point de vue Français, influençant également d'autres nations européennes. Elle causa elle aussi des difficultés en égyptologie, notamment entre Mariette et son collègue H. Brugsch. ${ }^{29}$ Ce sont néanmoins les Français et les Britanniques qui monopolisent les fouilles dans les années 1880-1890. Un tournant s'opère dans les années 1905-1907, avec l'arrivée d'importantes expéditions américaines et la création de l'institut allemand par Borchardt. ${ }^{30}$ Pour la période et le sujet qui nous concernent, la rivalité franco-britannique a donc un rôle plus important, mais l'Allemagne n'en est pas moins un acteur à prendre en compte. Tout ceci tend à montrer la forte interdépendance qui se créé, au tournant du siècle, entre égyptologie et la politique internationale.

C'est dans cette perspective que Maspero, dès son premier mandat à la tête du Service des Antiquités (1881-1886), avait fait prendre un nouveau tournant à l'archéologie égyptienne en autorisant les équipes étrangères à fouiller la Vallée du Nil - toujours sous l'autorité du Service des Antiquités, privilège jusqu'ici réservé aux équipes franco-égyptiennes, dirigées par Mariette et travaillant au nom de ce Service. ${ }^{31}$ Le contexte diplomatique est le premier motif qui pousse Maspero à agir ainsi : c'est tout d'abord un geste envers les Britanniques qui mettent sur pied l'EEF entre 1881 et 1882. Ceux-ci souhaitent venir fouiller en Egypte, et Maspero, voyant leur implication comme une aide bienvenue dans sa mission de protection du patrimoine égyptien, fait en sorte que l'autorisation de fouiller - et de recevoir en échange une partie du résultat de leurs fouilles - leur soit exceptionnellement accordée. En leur octroyant les moyens de mener à bien leurs travaux archéologiques sur le sol égyptien, sous le contrôle du Service des Antiquités, il joue leur jeu diplomatique et s'assure ainsi leur bienveillance envers le Service, afin de garantir la pérennité de sa direction française. ${ }^{32}$ Cette exception britannique ouvre, volontairement ou non, une brèche où s'engouffrent bientôt d'autres nations européennes. Outre l'enjeu diplomatique, Maspero lui-même avance deux raisons à ce qu'il considère comme un progrès du Service, dans sa préface aux Rapports sur le fonctionnement du Service des Antiquités de $1910:^{33}$ la première est, déjà à la fin du XIX ${ }^{\text {ème }}$ siècle, le caractère urgent des fouilles qu'on désignerait aujourd'hui comme d'archéologie préventive. En effet, la modification des techniques d'agriculture que connaît l'Egypte moderne, notamment en termes d'irrigation, engendre un processus d'expansion des terres cultivables qui met en danger son paysage archéologique. Il s'agit donc de le préserver, ou du moins de le fouiller avant qu'il ne soit détruit. De fait, l'ouverture des autorisations de fouille aux équipes étrangères permet un travail démultiplié en efficacité, mais aussi en moyens. Cela mène au second motif invoqué par le directeur : l'aspect financier fait là encore partie de l'équation. Souvenons-nous que la dette égyptienne est précisément la raison qui explique la tutelle britannique : il n'est donc pas étonnant de constater que le Service des Antiquités, depuis sa création par Mariette, pâtit, selon tous les rapports écrits par ses différents directeurs, d'un manque cruel de ressources financières face à l'immensité de la tâche à accomplir. Dès lors, inviter les équipes étrangères à participer aux fouilles, sous le contrôle du Service mais sur leurs propres deniers, est une façon pour Maspero d'économiser les ressources du Service sur les fouilles, afin de les consacrer aux travaux de conservation et de consolidation des monuments dégagés, comme il le souligne régulièrement dans ses rapports. Ce système est d'autant plus adapté que les lois de protection du patrimoine égyptien et du partage de fouilles garantit une part des découvertes au gouvernement égyptien, qui est le propriétaire officiel de tout objet trouvé sous son sol depuis $1891 .{ }^{34}$ Maspero peut ainsi se féliciter de ce que le « Musée s'est enrichi, sans bourse délier, d'une quantité de monuments précieux qu'il ne se serait pas procurés avec ses seules ressources $»{ }^{35}$

\section{Fonctionnement de la communauté égyptologique désormais internationale sous l'autorité du Service : entre collaboration et équilibre diplomatique précaire}

Durant cette période d'internationalisation du Service des Antiquités, ce dernier gère trois types de travaux : le premier, qui concentre la majorité de ses ressources financières propres, concerne les travaux de conservation et restauration des monuments, destinés à la fois à la sauvegarde du patrimoine historique et à 
assurer la sécurité des touristes les visitant, selon les écrits de Maspero. ${ }^{36}$ Si le Service peut se permettre de concentrer ainsi ses dépenses, c'est que les fouilles et sondages archéologiques proprement dits sont quant à eux exécutés - et financés - par les équipes étrangères grâce au nouveau système, après avoir obtenu un permis octroyé pour un site et une période donnés de la part du Service, qui les délivre en accord avec le
Comité d'Archéologie. Chaque année, c'est une vingtaine de sites en moyenne qui sont fouillés par les égyptologues non-membres du Service. Ainsi, sur la période 1903-1912, qui correspond aux dix premières années de fouilles de Schiaparelli, la répartition des sites selon les données fournies par les Rapports sur la marche du Service des Antiquités rédigés par Maspero correspond à celle qui suit (Document 1).

Document 1. Répartition des concessions de fouilles parmi les principales équipes étrangères, d'après les Rapports du Service des Antiquités, 1903-1912 37

\begin{tabular}{|c|c|c|c|c|c|c|c|}
\hline & $\begin{array}{c}\text { Italie (MAI, } \\
\text { fouilles de } \\
\text { Schiaparelli) }\end{array}$ & $\begin{array}{c}\text { France } \\
\text { (IFAO) }\end{array}$ & Royaume-Uni & Allemagne & Etats-Unis & Autres & Total \\
\hline 1903 & 4 & 6 & 7 & 3 & 1 & 0 & 21 \\
\hline 1904 & 3 & 3 & 6 & 1 & 2 & 0 & 15 \\
\hline 1905 & 4 & 5 & 7 & 2 & 2 & 0 & 20 \\
\hline 1906 & 2 & 5 & 9 & 5 & 3 & 0 & 24 \\
\hline 1907 & 0 & 4 & 7 & 3 & 4 & 3 & 21 \\
\hline 1908 & 2 & 7 & 4 & 5 & 4 & 1 & 23 \\
\hline 1909 & 1 & 4 & 5 & 5 & 4 & 0 & 19 \\
\hline 1910 & 1 & 4 & 6 & 1 & 6 & 1 & 19 \\
\hline 1911 & 3 & 4 & 7 & 2 & 5 & 1 & 22 \\
\hline 1912 & 2 & 4 & 6 & 4 & 6 & & 0 \\
\hline Total & 22 & 46 & 64 & 31 & 37 & 6 & 22 \\
\hline
\end{tabular}

La prépondérance des Britanniques parmi les équipes étrangères est bien visible : ce sont eux qui semblent les plus actifs, ou tout du moins ceux dont Maspero présente le plus de travaux dans ses rapports officiels. Que cette prédominance soit liée au nombre réel de concessions accordées ou à la surreprésentation de leurs activités par le directeur du Service sont deux hypothèses différentes mais qui mènent au même constat : une forme d'attention particulière accordée à ceux qui dirigent finalement l'Egypte et le Service. Il s'agit alors de comprendre les raisons de cette prédominance : s'agit-il d'une forme de favoritisme de la part du Service, qui impliquerait par exemple des refus de concessions à d'autres pays au profit du Royaume-Uni, ou est-ce simplement le résultat de demandes plus abondantes dues à une plus grande activité archéologique de leur part ? Cette dernière possibilité s'avère juste, tout en n'excluant pas totalement la première. En effet, il semble que les Anglais aient été particulièrement demandeurs, voire insistants : $\mathrm{Gady}^{38}$ rapporte ainsi que Lord Cromer a pu forcer la main du directeur du Service afin d'obtenir une concession dans la nécropole de Giza pour un membre du Parlement britannique, et que Petrie a longtemps insisté pour obtenir une concession à Saqqara - chantier par tradition réservé au Service- avant de finalement se la voir refusée en 1904, non sans avoir fait appel à Lord Cromer une fois encore. Ainsi, l'hypothèse du favoritisme n'est pas entièrement valide puisqu'on constate certains refus, mais elle reste un paramètre à prendre en compte, puisqu'il y a bien eu tentatives chez les Britanniques d'user de leur position politique. Le constat d'une demande plus accrue de leur part, comparé aux autres nations, reste manifeste, et s'explique là encore par la position géopolitique de la Grande-Bretagne vis-à-vis de l'Egypte. Son implication directe sur le territoire égyptien, couplée à un intérêt solidement ancré pour l'égyptologie - au même titre que la France et l'Allemagne - encourage les archéologues à s'y rendre pour fouiller. Les Français partagent ce double avantage, implication politique et tradition scientifique : il n'est donc pas étonnant qu'ils les suivent de près, tout en ne les 
égalant pas. ${ }^{39} \mathrm{Il}$ faut en effet garder à l'esprit qu'une partie des égyptologues français sont alors membres du Service, et ne sont donc pas considérés comme travaillant pour la France mais pour la Direction Générale des Antiquités, donc l'Egypte. De fait, les équipes sont considérées comme étrangères dans la mesure où elles ne sont pas financées par le Service, celui-ci ne faisant que superviser les fouilles, en accordant les concessions, en effectuant les contrôles par des inspecteurs pendant le chantier ainsi qu'à la fin, au moyen du partage des fouilles.

Cette mission d'inspection, de supervision et de contrôle administratif et scientifique de l'ensemble des travaux archéologiques sur le sol égyptien est la troisième et dernière mission du Service des Antiquités Egyptiennes. Pour ce faire, des inspecteurs en chef contrôlent respectivement leurs secteurs qui recoupent en général les régions de Haute, Moyenne et Basse Egypte, ainsi que les sites particulièrement riches en chantiers tels que Saqqara ou Karnak. Ces postes d'inspecteurs sont habilement répartis entre Britanniques et Français dès 1904, suite au statu quo établi par l'Entente Cordiale : Quibell se voit attribué le secteur de Saqqara et Carter celui de la Haute Egypte, tandis que Legrain règne sur Karnak. ${ }^{40}$ Ils produisent des rapports sur le déroulement des fouilles et leurs résultats, de sorte que le Service garde un contrôle total sur toute découverte ou potentiel problème. De plus, le directeur lui-même effectue des tournées d'inspection sur l'ensemble de la vallée du Nil. Le Service tente ainsi de jouer un rôle fédérateur en s'ouvrant à une perspective internationale tout en gardant un contrôle important. Ainsi, sous l'apparence d'une collaboration internationale, les rivalités entre puissances coloniales européennes continuent à influencer l'évolution de l'archéologie égyptienne.

Pour autant, cette ouverture du Service à l'international, si elle trouve ses racines dans les enjeux politiques, a bel et bien des conséquences épistémologiques sur le développement de l'égyptologie. En effet, de cette démultiplication des fouilles naît un véritable essor de l'archéologie égyptienne : plus elle est pratiquée, plus ses méthodes se systématisent et ses techniques se précisent. ${ }^{41}$ Dès lors, cette précision scientifique provoque des mutations non seule-

SERYICE DES ANTIQUITÉS

AUTORISATION DE FOUILLES

Fe soussigné, Directeur Général du Service des Antiquités, agissant en vertu des pouvoirs qui me sont délegués, autorise par la présente Nemsiom 2 pogfessum Schioparati à exécuter des fouilles scientifiques à Le Mathé des Himses (Eiven) dans les terrains appartenant à l'État, libres, non batis, non cultivés, non compris dans la zone militaire, cimetières, carrières, etc., et, en général, non affectés à un service public.

Les terrains sur lesquels porte l'autorisation comprennent $\mathrm{In}_{\mathbf{a}}$ ratui on Remin (Ehibes) -

Les fouilles seront faites par les soins et aux frais de Monomi4 grofesom Schimpanch pendant toute la durée du travail, en présence d'un délégué du Service

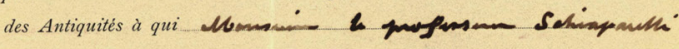
s'engage à payer la somme de 20 piastres tarif par jour à titre d'indemnité et les frais deproyage aller et retour.

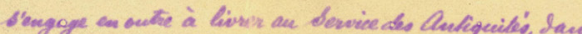

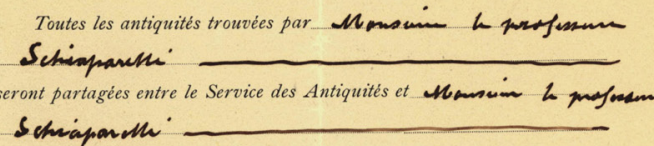
selon les règlements en vigueur.

Il est entendu, en outre, que Mansin a propsam Schimpanth. devra, une fois ses fouilles terminées, remettre dans un état satisfaisant tous les terrains sur lesquels il aura opéré; il ne sera autorisé à exporter les antiquités qui lui seront échues en partage que lorsqãe le Service des Antiquités aura constaté l'état satisfaisant de ces terrains. Mmanis Lquafessmon Schiopanthi s'engage à ne pas prendre d'estampage au papier humide sur les monuments coloriés et à déposer au Musée, et, si possible, à la Bibliothèque Khédiviale, un exemplaire des ouvrages, mémoires, tirages à part, recueils de gravures publiës par ses soins sur les objets découverts au cours de ses fouilles.

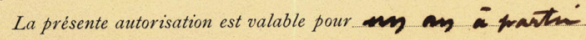

in 14 gamion 1908.
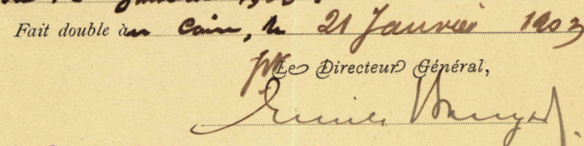

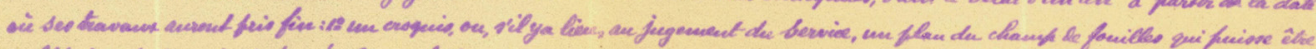

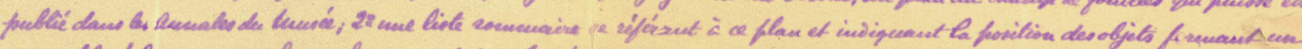

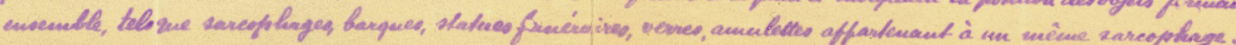

$$
\text { \& Bhipenth }
$$

Fig. 1. Document officiel du Service des Antiquités accordant la concession de fouilles à Schiaparelli pour la Vallée des Reines, en février 1903. Turin, Archivio di Stato, MAE, $2^{\circ}$ vers., M1 n6. Image utilisée avec autorisation. 
ment sur le terrain mais également dans les musées abritant des collections égyptiennes. L'importance de la provenance et du contexte archéologique devient peu à peu une évidence, ce qui engendre une nouvelle façon de penser la présentation mais aussi l'accroissement des collections. C'est de ce double contexte, politique et scientifique, qu'est née l'égyptologie italienne, sous l'impulsion d'Ernesto Schiaparelli, dans les premières années du XX⿳亠丷⿵冂丶 ${ }^{\text {è }}$ siècle (Fig. 1).

\section{L'internationalisation de l'égyptologie : un terreau favorable à l'Italie, et une opportunité saisie par Schiaparelli}

Ernesto Schiaparelli évolue en effet au contact de cette effervescence égyptologique dès la fin de ses études, en côtoyant les cours de Maspero lui-même, à Paris, entre 1877 et 1880. À l'issue de cette formation, il prend la tête de la section égyptienne du musée de Florence, avant de devenir, en 1894, directeur du Museo Egizio à Turin. Mais sa carrière ne se limite pas aux murs des musées italiens : Schiaparelli, dès le début de sa carrière, bénéficie de la nouvelle politique internationale de son ancien professeur désormais à la tête du Service des Antiquités Egyptiennes et intimement lié à la direction de l'Institut Français d'Archéologie Orientale. ${ }^{42}$ Ainsi, dès 1884 , Schiaparelli commence son expérience du terrain en visitant Bubastis, Akhmim puis Assouan, et s'illustre comme épigraphiste, travaillant principalement à relever des inscriptions. ${ }^{43}$ Ce rapport direct avec l'Egypte n'est pas sans lien avec sa carrière muséale, puisqu'il entreprend des campagnes d'acquisition d'objets sur place pour le musée de Florence. Il lie ainsi des contacts dans le pays, notamment avec des moines Franciscains présents au Caire et à Louqsor, qui s'avèrent plus tard d'une aide précieuse pour la gestion pratique des fouilles. ${ }^{44}$ S'il commence, de la même façon qu’à Florence, par acquérir des objets sur le marché de l'art en arrivant au musée de Turin, la concomitance de tous les éléments que nous avons pu évoquer ici le pousse rapidement à changer d'approche, et ce dès $1901 .{ }^{45}$ En effet, l'opportunité que représente ce mouvement d'ouverture de l'archéologie égyptienne à l'international, le changement épistémologique qu'il engendre en plaçant le contexte archéologique et la provenance des objets au premier plan, ainsi que le parcours et les contacts personnels de Schiaparelli, l'ayant placé à la croisé du terrain et des musées, influencent cette décision. Tous ces éléments menèrent ensemble le conservateur à considérer, pour la première fois en Italie, ${ }^{46}$ qu'entreprendre des fouilles archéologiques pour accroître les collections du Museo Egizio s'avérerait plus efficace, précis, et peut-être finalement moins cher que de s'en remettre au marché de l'art, qui encourageait les fouilles clandestines et présentait toujours le risque d'acquisition de faux. ${ }^{47}$

En effet, d'un point de vue scientifique et muséologique, cela devait d'abord permettre de compléter les lacunes, afin que le musée puisse couvrir toutes les périodes du prédynastique aux époques tardives l'Ancien Empire par exemple, était peu représenté dans les fonds antérieurs à 1903. Mieux encore, cela avait pour avantage d'approfondir l'étude de sites dont le musée possédait déjà des éléments, et de constituer des ensembles archéologiques cohérents, dont la présentation permettrait d'étudier les objets dans leur contexte, révélant ainsi tout leur intérêt historique, en tant qu'outil de compréhension de la civilisation égyptienne. Ce fut notamment le cas pour Deir el-Medina : la collection Drovetti ${ }^{48}$ comprenait en effet un nombre important d'objets provenant manifestement du site, bien que la provenance ait rarement été indiquée lors de l'acquisition. ${ }^{49}$ Schiaparelli le confirme dans son rapport de fouille : "Questa regione aveva per la nostra Missione particolare interesse (...) principalmente perché dalla medesima provenne indubbiamente parte importantissima, per non dire la parte maggiore, delle collezioni già possedute dal Museo di Torino $» .{ }^{50}$ L'exemple le plus parlant illustrant le succès de cette démarche est sans doute celui de la tombe de Kha : le fond Drovetti de 1824 comprenait une stèle funéraire de la XVIIIème dynastie ${ }^{51}$ dédiée à Kha, « Chef des travaux dans la Grande Place » soit architecte des tombes royales, et à son épouse Merit (Fig. 2).

Presque un siècle plus tard, en 1905, les fouilles de Schiaparelli dans la nécropole des artisans de Deir el-Medina ont livré non seulement la chapelle funéraire d'où provenait sans doute la stèle, mais le tombeau intact du couple, contenant l'ensemble de leur mobilier funéraire (Fig. 3). 


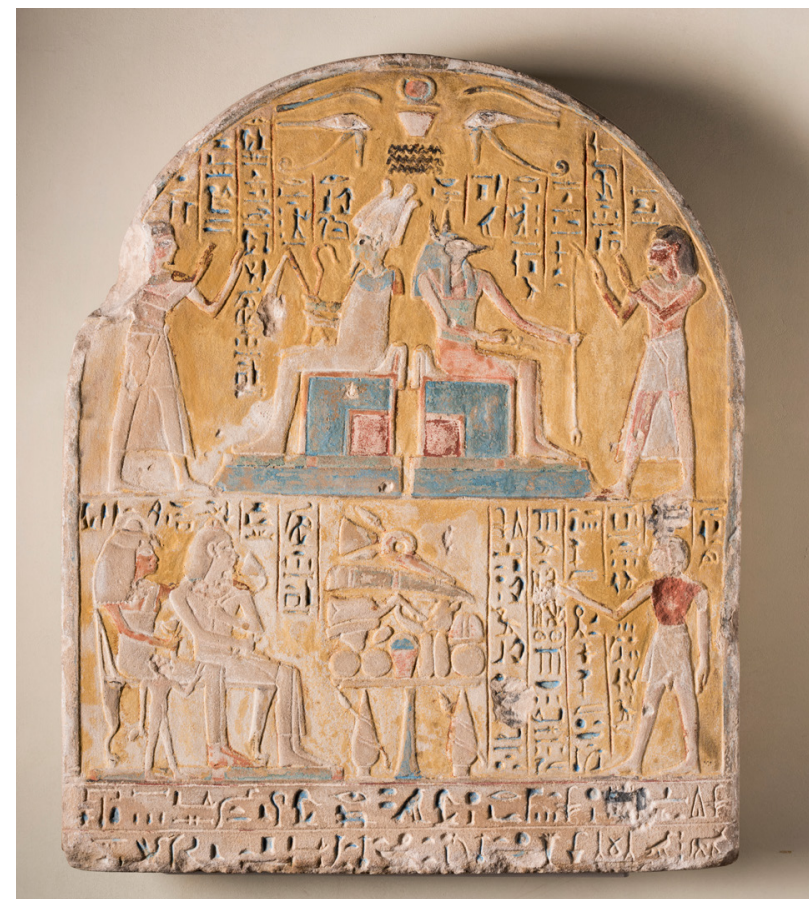

Fig. 2. Stèle funéraire de Kha et Merit, présente dans les collections du musée depuis l'acquisition du fond Drovetti en 1824. XVIIIİme dynastie (1539-1292 av. JC), grès, Museo Egizio di Torino, C. 1618.
Ces considérations scientifiques que l'on ne peut que constater a posteriori semblent s'inscrire dans un mouvement épistémologique plus large impliquant l'accroissement de l'importance du contexte archéologique dû à la multiplication des fouilles. Pour autant, les lettres adressées par Schiaparelli au Ministre de l'Instruction Publique (Fig. 4) afin de justifier son projet révèlent des arguments plus à mêmes de convaincre le gouvernement italien en rapportant à nouveau la question des fouilles à des considérations géopolitiques. En effet, la justification principale soulignée par l'égyptologue est celle $\mathrm{du}$ rayonnement international de l'Italie $:^{52}$ il pique l'attention du Ministre en soulignant que les principales nations européennes sont déjà présentes en Egypte, ${ }^{53}$ et en mettant en avant la glorieuse tradition scientifique italienne, ${ }^{54}$ ainsi que l'importance de l'étude de la civilisation égyptienne pour la connaissance historique de l'époque..$^{55}$ Pour mieux convaincre, il présente ce projet comme une oppor-

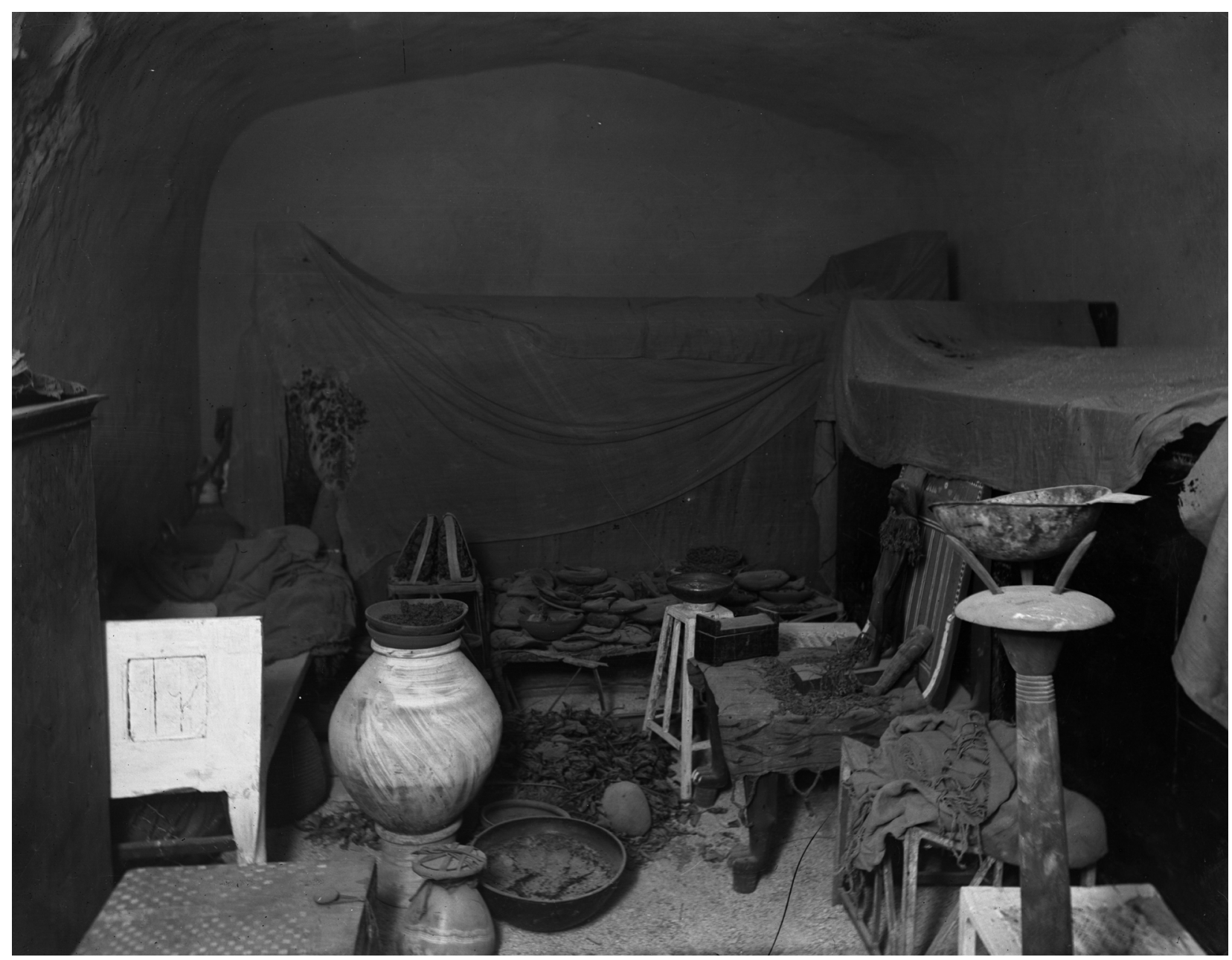

Fig. 3. Tombe de Kha et Merit telle qu'elle fut découverte par Schiaparelli et son équipe en 1905, soit presque un siècle après I'acquisition de leur stèle funéraire par le musée de Turin. Photographie d'archive du Museo Egizio di Torino, C. 2070. 
tunité à saisir puisqu'il parle tantôt d'une invitation, tantôt d'une proposition faite au Museo Egizio par Maspero, qui s'était pourtant contenté de répondre à une demande d'autorisation de fouilles formulée par Schiaparelli en $1902 .{ }^{56}$ Ce dernier ne manque pas de mentionner également l'apport de pièces que cela représenterait pour le musée, ainsi que l'implication financière selon lui tout à fait surmontable, ${ }^{57}$ sans pour autant qu'il ne désigne les fouilles comme moins chères que l'acquisition sur le marché de l'art.

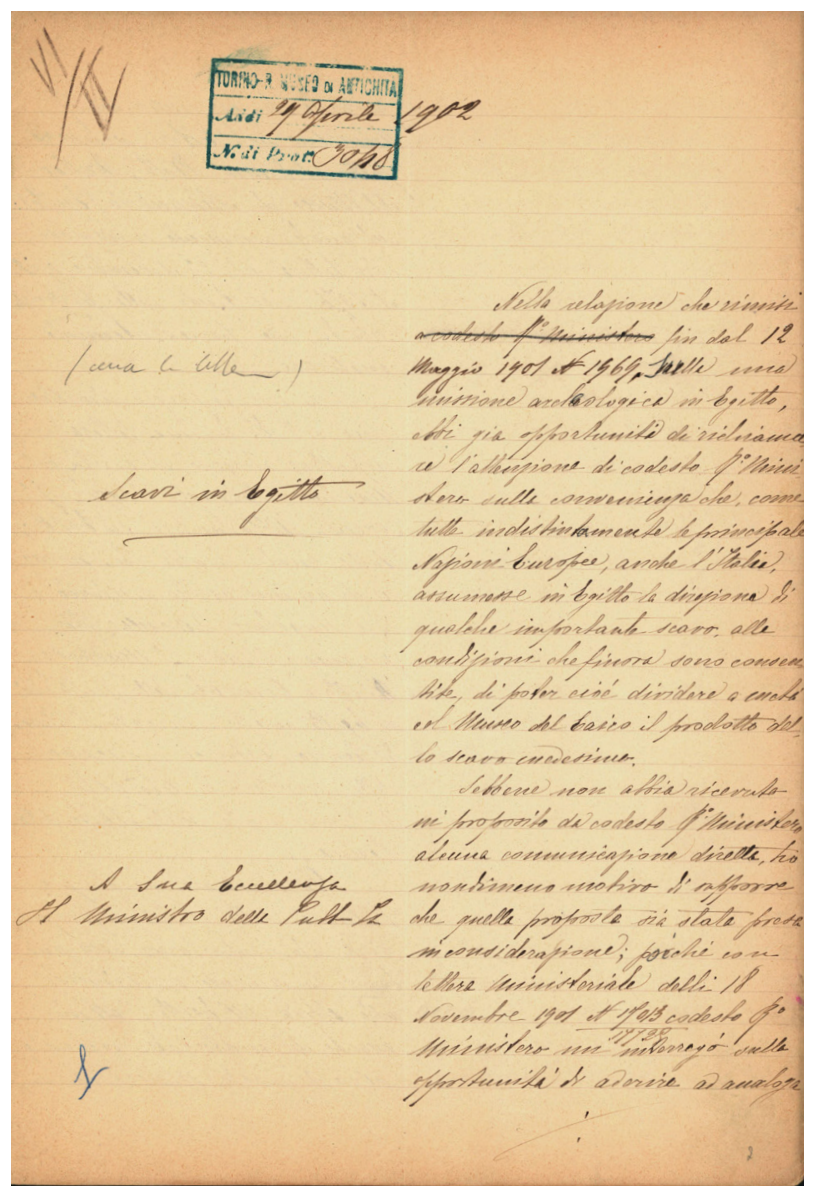

Fig. 4. Lettre de Schiaparelli au Ministère de I'Instruction Publique défendant la création d'une Mission Archéologique Italienne (MAl) en Egypte, datée du 29 avril 1902. Turin, Archivio di Stato, MAE, $2^{\circ}$ vers., M1 n5, Prot. 3048. Image utilisée avec autorisation.

Ainsi, ce changement d'approche introduit par Schiaparelli dans l'acquisition d'objets, issu d'une convergence de facteurs diplomatiques, épistémologiques et personnels, se sont révélés être un véritable tournant dans l'histoire du Museo Egizio : entre 1903 et 1920, ce sont treize campagnes de fouilles qui ont été menées dans onze sites différents ${ }^{58}$ par Schiaparelli et ses collaborateurs, apportant plus de 16
000 pièces aux collections. ${ }^{59}$ Plus encore que leur nombre, c'est leur valeur scientifique et la cohérence des ensembles ainsi constitués qui donnent au musée de Turin ses lettres de noblesses. La nouvelle muséologie du musée, rénové en 2015, se veut l'héritière de ce lien étroit entre musée et archéologie introduit par Schiaparelli : les ensembles d'objets sont présentés autant que possibles par sites, et des photographies d'archives évoquent aujourd'hui les fouilles et le contexte archéologique afin d'en souligner la provenance et la cohérence.

À plus large échelle, l'initiative de Schiaparelli, qui s'inscrit, on le voit, dans le contexte politique et scientifique de l'époque, marque un tournant dans l'histoire de l'égyptologie italienne, qui pour la première fois se constitue en une mission archéologique italienne officielle envoyée en Egypte, sur le terrain. En effet, afin de pouvoir entreprendre ses fouilles, Schiaparelli crée en 1903, avec l'appui financier du roi Victor Emmanuel III et des Ministères des Affaires Etrangères et de l'Instruction Publique, la MAI, ou Mission Archéologique Italienne. ${ }^{60}$ Maspero salue ainsi l'arrivée de l'Italie dans la vallée du Nil : "M. Schiaparelli avait une mission officielle du gouvernement italien. L'Italie s'est décidée en effet à suivre l'exemple que lui donnaient l'Allemagne, l'Angleterre et la France, et elle s'est associée à l'exploration archéologique de l'Egypte ».${ }^{61}$ Schiaparelli ouvre ainsi la voie, en saisissant l'opportunité offerte indirectement par les rivalités franco-anglaises, qui ont poussé le Service des Antiquités Egyptiennes à l'ouverture des fouilles de terrain aux autres puissances européennes, et de fait, aux Italiens. Cependant, bien que ces jeux diplomatiques imprégnant l'archéologie égyptienne aient pu créer une opportunité propice pour l'égyptologie italienne, ils peuvent parfois se révéler moins favorables. Dès 1903, Schiaparelli est confronté à des rivalités sur le terrain : à Giza d'abord, où il partage la concession avec les Allemands de l'Université de Leipzig menés par Steindorff, et l'équipe américaine de Reisner, ${ }^{62}$ mais aussi à Hermopolis Magna, où Breccia se heurte à d'autres égyptologues allemands, menés par Rubensohn. ${ }^{63}$ Le détail des différends n'est pas mentionné par la documentation officielle $:^{64}$ toujours est-il qu'en 1905, les Italiens abandonnent la concession d'Hermopolis aux Allemands, ce qui témoigne du poids qu’a pu avoir cette concurrence. 
Travaux scientifiques et discours officiel : le miroir déformant des enjeux diplomatiques?

Plus encore que ces escarmouches, ce qui frappe dans la place accordée aux Italiens au sein de la communauté égyptologique internationale par la Direction Générale des Antiquités, c'est le décalage entre l'importance des découvertes faites par l'équipe de Schiaparelli et leur poids dans les écrits officiels. En effet, dès 1904, les Italiens rencontrent un succès majeur dans la Vallée des Reines, marquant l'histoire des découvertes égyptologiques : la mise au jour de la tombe de Néfertari (QV66). Découverte en même temps que plusieurs tombes de princes du Nouvel Empire dont trois fils de Ramsès III, et bien que déjà pillée, elle se démarque non seulement par le statut de sa propriétaire, mais surtout par la qualité de ses peintures pariétales admirablement conservées. Que ces remarquables résultats trouvent très peu d'échos dans les publications du Service, qui se veulent pourtant références en termes d'informations officielles sur les dernières découvertes faites sur le sol égyptien, ${ }^{65}$ cela pose alors question.

En effet, la création des Annales du Services des Antiquités Egyptiennes par Victor Loret en $1900^{66}$ reflète l'effort fourni par le Service pour se positionner en tant qu'institution fédératrice et incontournable, et pour créer un lien entre les travaux des différents pays en permettant la publication des activités de toutes les équipes, celles du Service autant que les missions étrangères. Quand ce ne sont pas les égyptologues eux-mêmes qui y publient leurs derniers travaux, ce sont les inspecteurs des différents secteurs qui dressent chaque année le bilan des fouilles effectuées dans leur zone. Outre cette publication scientifique, Maspero en tant que directeur du Service rédige chaque année, de manière plus administrative, un Rapport sur le fonctionnement du Service des Antiquités de l'Egypte, dès son second mandat, en 1899. Or, du côté des Annales, la tombe QV66 n'est mentionnée qu'assez brièvement dans le rapport d'Howard Carter de 1905 sur les travaux effectués en Haute-Egypte en 1903-1904 : après avoir mentionné les noms des tombes découvertes, celle de Néfertari n'étant pas mise en avant par rapport à celles des divers princes, il se contente de citer les travaux effectués par le Service pour assurer la protection de ces tombes. ${ }^{67}$ Quant au rapport de Maspero, il est encore plus succinct : il mentionne uniquement et brièvement la tombe de Khaemouaset, fils de Ram-

Document 2. Les travaux effectifs de la MAl et leur écho dans les publications du Service des Antiquités, entre 1903 et 1912

\begin{tabular}{|c|c|c|c|}
\hline Année & $\begin{array}{l}\text { Liste des fouilles } \\
\text { italiennes }^{68}\end{array}$ & $\begin{array}{c}\text { Mentions des fouilles } \\
\text { italiennes dans les Rapports } \\
\text { du Service }\end{array}$ & $\begin{array}{l}\text { Mentions des fouilles } \\
\text { italiennes dans les ASAE }\end{array}$ \\
\hline 1903 & $\begin{array}{l}\text { Giza, Vallée des Reines, } \\
\text { Héliopolis, Hermopolis Magna }\end{array}$ & $\begin{array}{l}\text { Giza, Vallée des Reines, } \\
\text { Héliopolis, Hermopolis Magna }\end{array}$ & $\begin{array}{l}\text { Vallée des Reines (mention } \\
\text { succincte des tombes } \\
\text { de Khaemouaset et d'un autre } \\
\text { prince dans un rapport } \\
\text { de Carter) }\end{array}$ \\
\hline 1904 & $\begin{array}{l}\text { Vallée des Reines, Héliopolis, } \\
\text { Hermopolis Magna }\end{array}$ & $\begin{array}{c}\text { Hermopolis Magna, } \\
\text { Vallée des Reines, Héliopolis }\end{array}$ & - \\
\hline 1905 & $\begin{array}{c}\text { Vallée des Reines, } \\
\text { Deir el-Medina, Héliopolis, } \\
\text { Qaw el-Kebir, Badari, } \\
\text { el-Hammamiya }\end{array}$ & $\begin{array}{l}\text { Héliopolis, Vallée des Reines, } \\
\text { Deir el-Medina, } \\
\text { Gau (=Qaw el-Kebir), Badari } \\
\text { (comprenant Hammamiya) }\end{array}$ & $\begin{array}{c}\text { Vallée des Reines } \\
\text { (mention de la tombe } \\
\text { de Néfertari et de plusieurs } \\
\text { princes) }\end{array}$ \\
\hline 1906 & $\begin{array}{c}\text { Deir el-Medina, Héliopolis, } \\
\text { Qaw el-Kebir, Assiout, } \\
\text { el-Hammamiya }\end{array}$ & Deir el-Medina, Héliopolis & - \\
\hline 1907 & - & - & - \\
\hline 1908 & Deir el-Medina, Assiout & Deir el-Medina, Assiout & - \\
\hline 1909 & Hermopolis Magna & Deir el-Medina & - \\
\hline 1910 & Assiout, Gebelein, El Bahnasa & Assiout & - \\
\hline 1911 & Assiout, Gebelein, El Bahnasa & Gebelein, Assiout, El Bahnasa & - \\
\hline 1912 & Assiout, El Bahnasa & Gebelein, Assiout & - \\
\hline Total & $\begin{array}{l}29 \text { chantiers (tous les sites } \\
\text { et années cumulés) }\end{array}$ & $\begin{array}{l}24 \text { chantiers (tous les sites } \\
\text { et années cumulés) }\end{array}$ & \\
\hline
\end{tabular}


sès III dans son rapport de $1903 ;{ }^{69}$ le résumé de 1904 se borne à la phrase suivante : "À Thèbes, dans la Vallée des Reines, [les Italiens] ont vidé les tombeaux découverts l'an passé et ils nous les ont livrés : nous avons mis des portes aux principaux d'entre eux et nous les avons ouverts partiellement au public "; $7^{70}$ quant à celui de 1905, il ne mentionne pas plus la tombe de Néfertari découverte l'année précédente : «[Schiaparelli] a déblayé plusieurs tombeaux à la Vallée des Reines sans y rien rencontrer qui valût $"{ }^{71} \mathrm{On} \mathrm{ob}-$ serve le même phénomène pour l'autre importante découverte de la carrière de Schiaparelli, entre 1905 et 1906 : l'exploration de la tombe de l'architecte royal Kha, trouvée intacte dans la nécropole de Deir el-Medina, phénomène suffisamment rare pour être relevé, d'autant plus lorsque le mobilier funéraire est aussi riche et complet, et qu'en dépit des lois de partage des fouilles alors en vigueur, l'archéologue en a ramené l'intégralité au musée de Turin. Pour autant Maspero ne mentionne en 1905 que l'aspect « clément $~^{72}$ du site de Deir el-Medina envers la mission italienne, en évoquant la découverte d'archives de papyrus démotiques, et résume les fouilles du site en 1906 par la description suivante : " [Schiaparelli] a dégagé, à Dêîr-el-Médinéh, trois ou quatre tombeaux peu endommagés de la XXंme et de la XXİme dynastie (...) $»{ }^{73}$ Quant aux Annales $d u$ Service, elles n'en font aucune mention. Dans l'ensemble, pour les dix premières années de fouilles de Schiaparelli, on peut comparer la liste des travaux effectifs de la mission italienne et leurs répercussions dans les publications officielles du Service comme suit dans le Document 2. On constate que les échos dans les publications officielles sont lacunaires par rapports aux activités effectives de la Mission Archéologique Italienne.

A partir de ces deux constats, le décalage entre l'importance des découvertes italiennes et leur mention dans ces publications du Service d'une part, et les informations partielles sur les activités générales de la MAI d'autre part, il s'agit de formuler plusieurs hypothèses afin de comprendre ce phénomène qui semble pointer vers une sous-représentation des travaux italiens par le Service des Antiquités Egyptiennes. La première consiste à interroger la nature des publications étudiées pour s'assurer que les fouilles étrangères y auraient bien leur place. Si l'on prend les Annales (ASAE), on peut ainsi supposer que l'absence italienne puisse être due au fait que seuls les membres du Service y publient des articles. C'est en effet ce que suggère Maspero lors du lancement de ce périodique, puisqu'il en parle comme d'une nouvelle revue égyptologique "dont les savants attachés au Musée de Giza [doivent] être les rédacteurs attitrés $»{ }^{74}$ Le musée étant une composante du Service des Antiquités, on peut en déduire qu'il s'agit ici plus largement des membres de ce Service, travaillant donc au nom du gouvernement égyptien. Si c'était bien l'intention initiale, l'analyse des ASAE - sur la période des dix premières années de fouilles de Schiaparelli - montre que la réalité est plus nuancée. On considère ici comme membres du Service les savants travaillant pour celui-ci à la période de rédaction de ces numéros des ASAE (Document 3). On constate que si une légère majorité des auteurs participe en effet à cette revue au titre de membre

Document 3. Statut des auteurs des ASAE (1903-1912) ${ }^{75}$

\begin{tabular}{|c|c|c|c|}
\hline Numéros ASAE & Nombre total d'auteurs & Auteurs membres du Service & Auteurs non-membres \\
\hline 1903 (no. 4) & 16 & 9 & 7 \\
\hline 1904 (no. 5) & 17 & 7 & 10 \\
\hline 1905 (no. 6) & 17 & 6 & 11 \\
\hline 1906 (no. 7) & 26 & 17 & 9 \\
\hline 1907 (no. 8) & 20 & 9 & 8 \\
\hline 1908 (no. 9) & 17 & Pas de numéro & 9 \\
\hline 1909 & Pas de numéro & 8 & 7 \\
\hline 1910 (no. 10) & 17 & 10 & 6 \\
\hline 1911 (no. 11) & 17 & 7 & $47.5 \%$ \\
\hline 1912 (no. 12) & 13 & $52.5 \%$ & numéro \\
\hline Proportions sur la période & $100 \%$ & & \\
\hline
\end{tabular}


du Service des Antiquités de l'Egypte, $47.5 \%$ des auteurs y contribuent malgré tout en tant que chercheur " invité ", autrement dit, en tant qu'égyptologue travaillant pour une mission étrangère. Parmi ces contributeurs étrangers, on note une majorité de savants français (39.47\%), suivi de près par les Britanniques (30.26\%). La part des auteurs italiens sur la période vient en dernier, après les Allemands et les Américains, ${ }^{76}$ avec une proportion de $3.95 \%$, principalement grâce aux articles de Breccia, directeur du musée d'Alexandrie et non membre de la MAI. Par conséquent, des rapports de fouille par Schiaparelli ou un membre de la MAI auraient tout à fait eu leur place dans ces annales, et l'on peut d'ailleurs noter l'unique contribution de l'Italien, en 1921, dans laquelle il dresse un bilan des fouilles italiennes à Gebelein. ${ }^{77}$ Par ailleurs, les travaux italiens pourraient faire l'objet d'articles de la part des inspecteurs du Service des Antiquités responsables de leur secteur de fouilles, comme c'est le cas pour certains chantiers. Quant aux rapports administratifs du Service rédigés par Maspero, aucune différence particulière n'y est faite entre l'évocation des travaux étrangers et ceux du Service, ce dernier étant traité comme une Mission parmi d'autres.

Si les missions étrangères font bien partie de ces publications, quelle est donc la place de l'Italie parmi elles ? Le point de vue qualitatif donné par Maspero dans ses Rapports aurait tendance à suggérer un étonnant désintérêt pour la Mission italienne, puisqu'il se montre relativement laconique et finalement peu élogieux envers ses travaux pourtant remarquablement productifs : "M. Schiaparelli a passé aux bords du Nil presque sans s'y arrêter, et les quelques coups de pioche qu'il a donnés sur les sites concédés au Gouvernement italien ont été peu fructueux " $7^{78}$ "L'Italie, qui détient beaucoup de localités intéressantes, n'a utilisé que deux d'entre elles $» .{ }^{79} \mathrm{D}$ 'un point de vue plus quantitatif, les deux publications officielles du Service, l'une administrative, l'autre scientifique, mentionnent les travaux des différentes missions étrangères selon la répartition suivante.

Les données présentées ci-dessus concernant les Rapports du Service des Antiquités reprennent les valeurs présentées précédemment dans le Document $1^{80}$ sous forme de pourcentage, ce qui permet de constater la répartition par pays des fouilles
Document 4. Mentions des fouilles étrangères en Egypte dans les publications officielles du Service des Antiquités, $1903-1912^{81}$
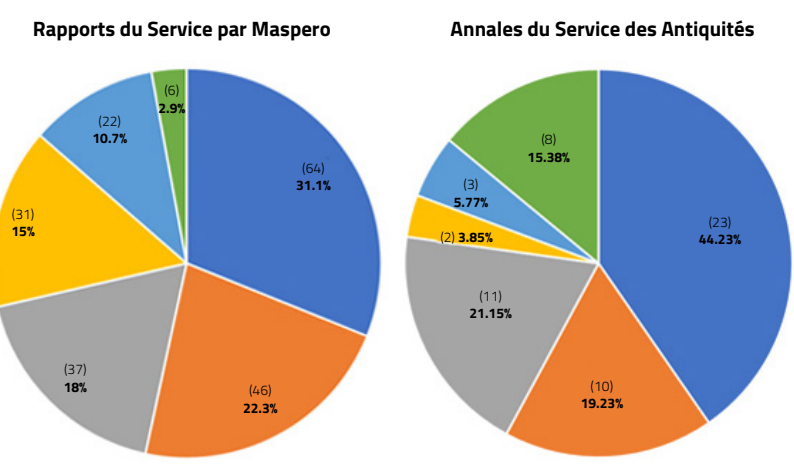

- Britanniques = Français = Américains = Allemands = Italiens | Autres (8) Valeurs absolues

étrangères citées par Maspero, qui en compte 206 au total dans ses Rapports entre 1903 et 1912. Quant aux Annales du Service des Antiquités, parmi les 111 mentions de fouilles effectuées en Egypte sur la période étudiée, un peu plus de la moitié est dédiée aux travaux du Service lui-même (53.1\%), réduisant le nombre de fouilles étrangères citées à 52 . C'est sur ce total que sont calculées les proportions par nation fournies ici. Les résultats concernant les deux publications mènent à première vue aux mêmes conclusions : une confirmation de la prévalence des travaux britanniques, suivis par les fouilles françaises légèrement en retrait, puisqu'une grande partie des Français travaille au nom du Service des Antiquités et non de la France. ${ }^{82}$ La prédominance de ces deux nations dans les publications paraît assez proportionnelle au nombre important de travaux qu'elles mènent, se distinguant ainsi largement des autres. Mais il est intéressant de noter le cas de l'Italie, par ailleurs semblable à celui de l'Allemagne : les travaux de ces pays sont bien moins souvent publiés scientifiquement, dans les ASAE, qu'ils ne sont administrativement cités dans les Rapports. Ainsi, l'Italie détiendrait $10.7 \%$ des fouilles menées par des équipes étrangères mais seuls $5.7 \%$ des articles concernant des fouilles étrangères dans les ASAE concernent des travaux italiens, presque moitié moins. De même, l'Allemagne effectuerait $15 \%$ des fouilles étrangères, tandis que ces travaux représentent moins de $4 \%$ des articles des ASAE traitant des chantiers menés par les étrangers, une sous-représentation toute aussi frappante, voire plus encore, que celle de l'Italie. On note pourtant de nombreux articles en allemand ou par des Al- 
lemands dans les ASAE sur cette période, mais pour beaucoup, ils ne traitent pas de rapports fouilles mais d'autres aspects de l'égyptologie, tels que la philologie ou des sujets d'études historiques. Cela peut se justifier par le fait que, bien que l'Allemagne soit reconnue pour l'excellence de ses universités et ait dominé la philologie dans le domaine de l'égyptologie, elle est peu réapparue sur le terrain de fouilles entre l'expédition Lepsius des années 1840 et l'ouverture du Kaiserlich Deutsche Institut für Ägyptische Altertumskunde, l'institut d'égyptologie allemand dirigé par Borchardt ${ }^{83}$ en 1904. Et ce retour fut de courte durée puisque dès 1914, la guerre et le scandale du buste de Néfertiti viennent de nouveau envenimer la situation. ${ }^{84}$ Bien qu'il y ait eu entre temps des noms importants, comme Ebers, Dümichen, H. Brugsch, ce retrait peut expliquer la faible représentation de l'Allemagne dans les rapports de fouilles publiés par les ASAE. Quoi qu'il en soit, il semble que, proportionnellement au nombre de chantiers effectifs de chacun, le Service tend à publier scientifiquement un plus grand nombre des travaux britanniques ou français que des travaux allemands ou italiens. Il y aurait donc moins un biais anti-italien qu'une tendance à mettre en avant les activités des deux nations qui sont politiquement impliquées dans la gestion de l'Egypte et de l'égyptologie. La part importante de la catégorie « autres » dans les Annales peut surprendre, mais tend finalement à confirmer cette idée : on a pris en compte sous cette dénomination les quelques fouilles Austro-hongroises, ${ }^{85}$ ainsi que des mentions de fouilles isolées de particuliers, Espagnol, Roumain, ou Egyptiens. Celles-ci sont rapportées par les membres du Service des Antiquités, qui ont pour mission de les superviser. Si elles arrivent ainsi, en quatrième position des travaux mentionnés par les Annales, alors même qu'elles sont ponctuelles et isolées, c'est peut-être là encore un reflet de la prédominance des activités des pays gestionnaires de l'archéologie égyptienne dans le discours officiel. Pour autant, cette théorie qui montre ainsi que l'Italie pâtit d'un manque général d'attention dans les publications officielles au même titre que l'Allemagne, en raison d'une focalisation sur les Britanniques et les Français due à leur implication politique en Egypte, se voit nuancée par la relative neutralité que l'on peut observer vis-à-vis des Etats-Unis : ils semblent repré- sentés de manière cohérente dans les publications officielles et ne rentrent ainsi ni dans un groupe ni dans l'autre.

Une dernière explication peut alors justifier cette place restreinte accordée aux travaux italiens dans les publications du Service : le manque de sources. Pour rapporter les résultats de la MAI, il est nécessaire qu'un de ses membres écrive un article à publier, ou qu'il fournisse au Service des Antiquités les documents nécessaires pour le faire. Or plusieurs éléments semblent indiquer que ce n'était pas le cas : Schiaparelli a uniquement publié les rapports de fouilles de la Vallée des Reines ${ }^{86}$ et de la tombe de Kha, ${ }^{87}$ ses deux grandes découvertes, et ne l'a fait qu’à partir de 1923, soit presque vingt ans plus tard, un retard important qu'il sent nécessaire de justifier dans la préface du premier rapport. ${ }^{88}$ Par ailleurs, il reçoit en 1922, au même titre que tous les égyptologues étrangers, une lettre d'avertissement du Service des Antiquités ${ }^{89}$ rappelant l'obligation pour les bénéficiaires de concessions de remettre certains documents au Service en fin de fouille : plan du site, liste d'objets découverts, rapport sommaire (Fig. 5). Plus encore, cette lettre rappelle que ces derniers ne dispensent pas le fouilleur de son devoir de publication, qu'il doit prendre en compte dans ses dépenses au même titre que les frais de fouilles plutôt que de consacrer toutes ses ressources au chantier proprement dit. Les publications scientifiques sont donc exigées, et leur absence doit être justifiée auprès du Service, sous peine de suppression de la concession accordée. L'émission d'un tel document de rappel laisse donc entendre que ces règles n'étaient pas suffisamment suivies, et en l'absence de trace connue de tels documents émis par Schiaparelli, il est raisonnable de penser que les Italiens aient pu avoir des retards voire des lacunes dans leur transmission des bilans de fouilles au Service, de même que dans la publication de leurs travaux. Or, le devoir de publication est également un droit du fouilleur, qui bénéficie d'une forme de monopole pendant cinq années durant lesquelles " nul ne pourra traiter avant lui de la matière qu'il devra recouvrir, que celle-ci concerne l'ensemble de la fouille, une partie seulement ou même uniquement un de ses aspects $" .{ }^{90}$ Ce monopole, conjugué au retard de publication de la mission italienne, et au fait qu'elle n'ait peut-être pas toujours remis les 
documents post-fouilles requis, peut expliquer l'absence des travaux italiens des publications scientifiques du Service des Antiquités, qui peut ainsi être due à la gestion post-fouilles de Schiaparelli et de son équipe. En effet, il ne semble pas que l'archéologue italien ait publié dans des revues d'égyptologie plus locales, en Italie; peu d'entre elles étaient déjà fondées. Aegyptus, revue italienne d'égyptologie et de papyrologie fondée à Milan à la fin de sa carrière, en 1920, n'a pu publier qu'un article d'hommage lors du décès de l'égyptologue, ${ }^{91}$ un successeur de Schiaparelli en revanche, Giulio Farina, y a publié un rapport de mission de la MAI menée à Gebelein en $1930 .{ }^{92}$

Quant au silence concernant les importantes découvertes italiennes, il est comparable aux cas allemand et britannique. Etonnamment, la découverte du buste de Néfertiti par Borchardt à Amarna en 1912 puis celle de la tombe de Toutankhamon par Carter en 1922 ne sont pas plus mentionnées dans les publications du Service. Dans les trois cas, il s'agit de découvertes menant à un partage de fouilles délicat car relevant de pièces précieuses : la discrétion peut donc s'expliquer par le désir d'éviter toute polémique. Ainsi, Maspero ayant laissé, de manière encore inexpliquée, l'ensemble du mobilier de la tombe intacte de Kha au musée de Turin ${ }^{93}$ à une période où le système de partage avec le Service prévaut, a pu vouloir diminuer l'impact de la découverte pour ne pas avoir à s'en justifier.

En somme, l'étude des publications officielles du Service des Antiquités montre que les travaux italiens en Egypte auraient bien leur place, au même titre que d'autres missions étrangères au sein des rapports administratifs autant que des annales scientifiques. Pourtant, l'évocation de leurs activités est non seulement lacunaire et parfois peu élogieuse, mais occupe en plus une part très restreinte, y compris proportionnellement aux nombres de travaux de chacun. Deux explications se dévoilent alors, sans nécessairement s'exclure l'une et l'autre. D'une part, un manque de rigueur de la MAI vis-à-vis de la publication de ses travaux, rendant difficile toute publication par le Service. D’autre part, un possible biais du discours officiel, qui ne semble pas spécifiquement tourné contre les Italiens, mais paraît plutôt être une tendance à mettre en avant les fouilles des pays

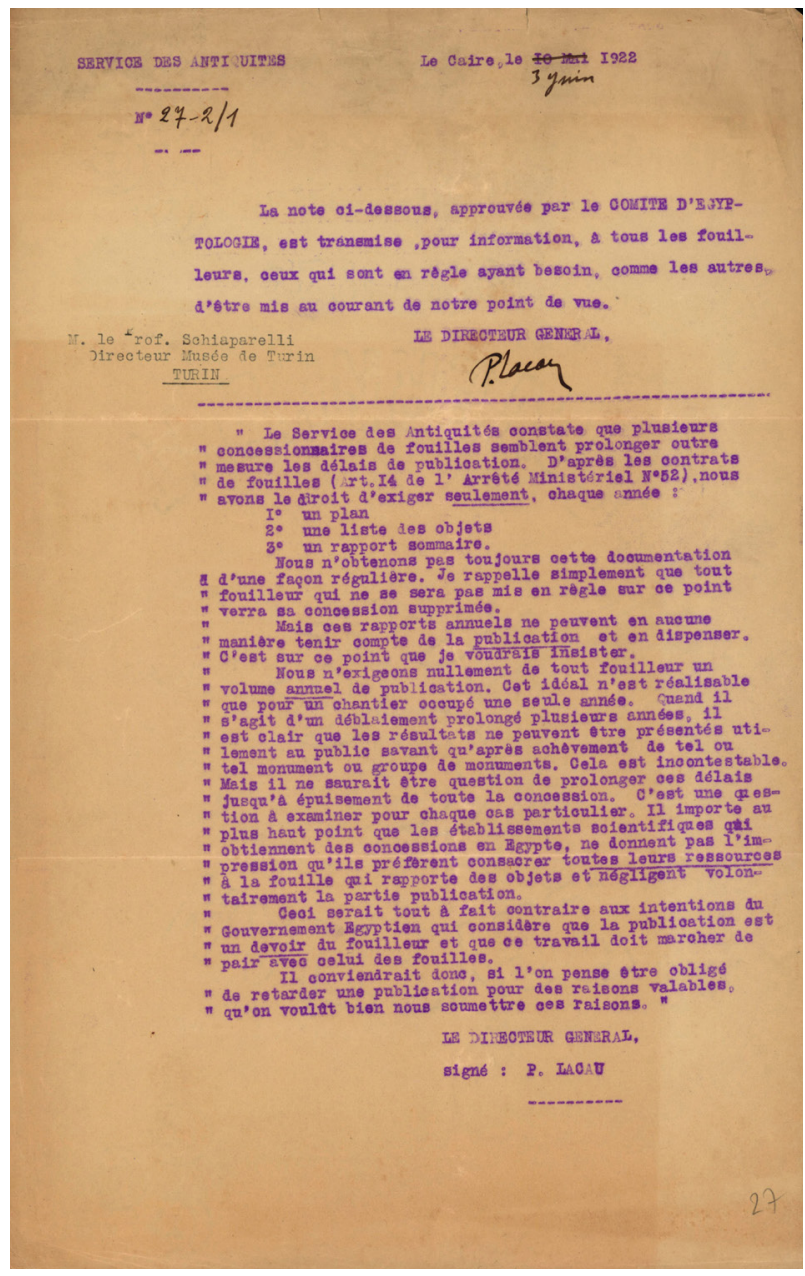

Fig. 5. Lettre du Directeur du Service des Antiquités Pierre Lacau, adressée "à tous les fouilleurs" en 1922, rappelant les obligations de ces derniers en échange de l'accord d'une concession de fouilles : la remise de documents de bilan en fin de fouilles, ainsi que le devoir de publication scientifique de leurs découvertes. Exemplaire reçu par Ernesto Schiaparelli. Datée du 3 juin 1922. Turin, Archivio di Stato, MAE, $2^{\circ}$ vers., M1 n21. Image utilisée avec autorisation.

prenant part à la gestion politique et scientifique de l'Egypte : la Grande-Bretagne et la France. Cela peut alors être interprété comme une conséquence du poids des enjeux diplomatiques sur la gestion de l'égyptologie : la rivalité des deux puissances coloniales vis-à-vis de l'Egypte pourrait ainsi expliquer leur prédominance dans le paysage administratif et officiel de l'égyptologie, les uns et les autres tentant de maintenir l'équilibre fragile de l'Entente Cordiale.

\section{Considérations finales}

Ainsi, l'impact des enjeux diplomatiques sur le développement de l'égyptologie est perceptible à deux niveaux. Cela a pu créer une opportunité favorable dont l'Italie a su tirer parti pour s'illustrer sur le ter- 
rain archéologique égyptien, par le biais de l'internationalisation du Service des Antiquités de l'Egypte provoqué par les rivalités franco-britanniques. Néanmoins, ce poids du contexte géopolitique influence également les discours et documents officiels, biaisant parfois la représentation des travaux scientifiques effectués. Dans un sens comme dans l'autre, l'étude de l'histoire de l'égyptologie apparaît comme indissociable de celle des rapports de

\section{Notes}

${ }^{1}$ Bednarski, dans Shaw et Bloxam (éd.), The Oxford Handbook of Egyptology.

${ }^{2}$ Del Vesco et Moiso (éd.), Missione Egitto.

${ }^{3}$ Sur ce point, voir par exemple Stevenson, dans Piacentini (éd.), Forming Material Egypt. Conservatrice au Petrie Museum de Londres, Alice Stevenson a montré à travers de multiples publications l'importance, pour les musées conservant des collections égyptiennes, de la contextualisation historique de leur découverte et de leur arrivée au musée. Ce projet a donné lieu à une publication visant à illustrer les histoires, anciennes et modernes, portées par de nombreuses pièces des collections égyptiennes du Petrie Museum : Stevenson, The Petrie Museum of Egyptian Archaeology, Characters and Collections.

${ }^{4}$ L'Italie toute récemment unifiée se lance dans l'expansion coloniale pour des raisons de politique interne plus qu'externe : il s'agit entre autre de mettre en avant une idéologie visant à renforcer le sentiment d'unité nationale, ainsi que d'endiguer l'émigration massive italienne du début du siècle. La jeunesse du pays explique une expansion plus modeste que ses voisins. Après avoir échoué à s'approprier la Tunisie, que s'accapare la France en 1881 par le traité du Bardo, l'Italie fait une tentative éphémère en Erythrée et Somalie dans les années 1890. C'est en Libye que les opérations sont finalement les plus fructueuses, après une pénétration pacifique entamée dès les années 1880, la guerre de 1911 livre la Cyrénaïque ainsi que la Tripolitaine à l'Italie. Outre les aspects purement militaire et politique, les ressortissants italiens sont présents un peu partout en Afrique du Nord, y compris en Tunisie, et en Egypte. Voir Miège, L'Impérialisme colonial italien.

${ }^{5}$ Il faut d'abord noter que l'Italie est représentée dans les deux commissions internationales concernant le redressement de la dette publique égyptienne, en 1876 et 1879 (voir De Gayffier-Bonneville, Histoire de l'Egypte moderne, p. 136-37.) Par ailleurs, les Italiens ont joué un rôle important dans la création de l'Université du Caire, où ils sont nombreux à enseigner jusqu'en 1911, lorsque l'invasion italienne en Libye compromet la relation avec l'Egypte. Voir sur cette question Reid, Cairo University and the Making of Modern Egypt, p. 38. Plus largement, l'influence culturelle italienne en Egypte est largement diffusée au XIX ${ }^{\text {ème }}$ forces internationaux dans lesquels s'inscrivent ses acteurs. Le cas italien ici présenté mène alors à nuancer l'image d'une archéologie égyptienne qui serait une paisible collaboration internationale, et parviendrait, contrairement à l'archéologie grecque, à « surpasser les divisions nationales ». ${ }^{94}$ Lâge d'or de l'égyptologie connu par Schiaparelli, se révèle être traversé, voire façonné, par les rivalités coloniales et les nationalismes propres au tournant du siècle.

siècle, avant d'être remplacée par celle du RoyaumeUni et de la France à partir de la fin des années 1860. L'Italien est la première langue parlée dans les ports méditerranéens au XIX ${ }^{\text {ème }}$ siècle, et l'une des principales langues pour la diplomatie, Mehemet Ali s'entourant de nombreux conseillers de ce pays (Reid, Cairo University and the Making of Modern Egypt, p. 39). C'est la presse italianophone qui domine l'Egypte au milieu du siècle, Lo Spettatore Egiziano devenant le premier journal égyptien notable en 1845, suivi par plusieurs dans les années 1850 . C'est également une compagnie italienne qui gère le service postal égyptien, ainsi que certains services sanitaires ou statistiques. Pas moins de 34926 immigrés italiens vivent en Egypte en 1907. Voir Reid, Whose Pharahos ?, p. 116, ainsi que p. 198.

${ }^{6}$ Il faut ici nuancer : Schiaparelli n'est pas le premier égyptologue italien en Egypte, mais le premier à fouiller au nom de l'Italie en tant qu'Etat-Nation. Avant cela, on compte de nombreux binômes franco-italiens collaborant sur différents projets : Rosellini et Champollion, Vassalli et Mariette, Barsanti et Maspero. Excepté Rosellini, ils travaillent au nom du Service des Antiquités, et non de l'Italie, tout comme les conservateurs dirigeant les musées égyptiens : Barsanti à Boulaq de 1891 à 1917, Botti en 1892 puis Breccia en 1904 au Musée d'Alexandrie. Reid dans Whose Pharaohs ?, p. 161 parle d'ailleurs du Musée Greco-Romain comme d'une enclave culturelle italienne dans l'Egypte colonisée. On peut également citer Belzoni et Drovetti dans les années 1820, qui étaient cependant des collectionneurs et non des égyptologues. Sur ce sujet, voir Bonacasa, dans La Rosa (éd.), L'archeologia italiana nel Mediterraneo fino alla seconda guerra mondiale, p. 46-47.

${ }^{7}$ Jason Thompson a ainsi intitulé le second volume de son histoire de l'égyptologie, Wonderful Things, II, The Golden Age : 1881-1914. Dans le premier chapitre de cette publication, il justifie ce titre comme suit : « [this period] was one of great discoveries, important institutional developments, and leaps in knowledge so that it has been termed 'the Golden Age of Egyptology' » (p. 5). Pour autant, il faut souligner que cet âge d'or est celui d'une égyptologie européenne, et que les égyptologues égyptiens ont pu contester une telle vision de l'archéologie égyptienne. Sur ce point, voir Reid dans Jankowski et Gershani (éd.), Rethinking Nationalism, ou plus récemment, de Reid 
également, Contesting Antiquity in Egypt.

${ }^{8}$ Sur la révolte d'Arabi Pacha et la situation économique et politique de l'Egypte à la fin du XIX'̀me siècle, voir Reid, dans Daly (éd.), The Cambridge History of Egypt, II, et plus récemment De Gayffier-Bonneville, Histoire de l'Egypte moderne.

${ }_{9}^{9}$ Goyon, Kyphi 5, (2006). Il faut noter que le Service des Antiquités Egyptiennes dépendait, à sa création, directement du Khédive. Néanmoins, en 1878, la France et le Royaume-Uni placent des représentants au gouvernement égyptien afin de protéger leurs intérêts : les Finances reviennent ainsi au Britannique Wilson, tandis que les Travaux Publics sont confiés au Français Blignières. Le Service est alors rattaché à ce dernier ministère, et le reste malgré le passage de l'ensemble du gouvernement égyptien sous domination britannique. Ce n'est qu'en 1929 qu'il sera plus logiquement rattaché au Ministère de l'Instruction Publique.

${ }^{10}$ Voir Khater, Le régime juridique des fouilles et des antiquités en Égypte, p. 76.

${ }^{11}$ Le projet de l'égyptologue Auguste Mariette a pu voir le jour grâce à l'implication de ses amis et collègues évoluant dans l'entourage du Vice-roi égyptien, Barthélémy de Saint-Hilaire et Ferdinand de Lesseps (le bâtisseur du canal de Suez).

${ }^{12}$ Voir n. 9.

${ }^{13}$ Tout d'abord, le brouillon du projet initial de Mariette ne contient pas une seule occurrence des mots « France » ou " français », voir l'article d'Elisabeth David, RdE 61 (2010). D'autre part, lors des premières discussions avec le gouvernement égyptien, Mariette, Lesseps et Saint-Hilaire sont d'accord pour ne pas mentionner immédiatement le projet de musée, allant pourtant de pair avec le Service, car cela pourrait contrarier le gouvernement français qui y verrait dès lors un risque pour le musée du Louvre et ses intérêts. Voir Gady, « Le pharaon, l'égyptologue et le diplomate », p. 217.

${ }^{14}$ Voir Gady, « Le pharaon, l'égyptologue et le diplomate », p. 220.

${ }^{15}$ On peut voir dans la création de la Mission Archéologique Française du Caire, confiée à Maspero en 1881, une façon pour le gouvernement français de s'assurer de garder un pied en Egypte, au cas où la succession de Mariette n'irait pas dans son sens.

${ }^{16}$ Voir Gady, « Le pharaon, l'égyptologue et le diplomate », p. 325-30.

${ }^{17}$ De nombreux écrits français relaient l'idée, depuis Champollion, que l'égyptologie serait une science française. Maspero va jusqu’à parler de l'égyptologie comme d'une « sorte de province de la science française » lors d'une session à l'Académie des Inscriptions et Belles-Lettres en 1909. Voir Gady, Les nouvelles de l'archéologie 126 (2011), p. 5. Cette idée trouve son apogée dans une publication par Maspero en 1915, « Les études égyptologiques », chapitre du livre La Science Française, édité par Lucien Poincaré à l'occasion de l'Exposition universelle de San Francisco, où la France présente une bibliothèque symbolique de toutes les sciences développées par la France. Une instrumentalisation nationaliste de l'égyptologie qu'il faut néanmoins replacer dans le contexte de la Première guerre mondiale et des Expositions universelles, exacerbant le sentiment national.

${ }^{18}$ Cette ambiguïté se perçoit à nouveau nettement dans les conflits créés autour du partage des fouilles entre Lacau, directeur français du Service, et Carter, égyptologue anglais, lors de la découverte de la tombe intacte de Toutankhamon en 1922. Alors que Lacau veut appliquer la nouvelle législation restrictive empêchant les objets de quitter l'Egypte, défendant ainsi les intérêts du pays, Carter et l'égyptologie anglaise le perçoivent comme une attaque française contre eux.

${ }^{19}$ Sur ce point, voir Piacentini (éd.), Victor Loret in Egypt (1881-1899).

20 « Voilà le corps diplomatique entier conquis au musée : quel changement de la position que m'avait légué Mariette à celle que je lèguerai à mon successeur ! Il est vrai que je perds quelques journées par an à des actes de politesses parfois ennuyeux (...) ». Maspero, lettre datée du 2 Mars 1886, dans David (éd.), Gaston Maspero, p. 172.

${ }^{21}$ Gady, « Le pharaon, l'égyptologue et le diplomate », p. 357, cite la lettre de Maspero au Ministère des Affaires Etrangères, datée du 24 septembre 1882.

22 « J'étais entre Rousseau et Mazuc qui m’ont donné des détails navrants sur le désordre que ces malheureux Anglais introduisent partout. Ils apportent leurs manières brutales de l'Inde, se font rembarrer même par les Arabes, et ne comprennent pas que, dans un pays habitué depuis soixante ans à l'administration française, on ne peut pas du jour au lendemain introduire les principes bizarres de la bureaucratie anglaise ». Maspero, lettre datée du 17 janvier 1884, dans David (éd.), Gaston Maspero, p. 55.

${ }^{23}$ Sur l'aide apporté à l'EEF, voir Gady, « Le pharaon, l'égyptologue et le diplomate », p. 360.

${ }^{24}$ Gady, Les nouvelles de l'archéologie 126 (2011).

${ }^{25}$ Traité de l'Entente Cordiale, Londres, 8 avril 1904.

${ }^{26}$ Maspero, lettre datée du 30 juin 1904, David (éd.), Gaston Maspero, p. 333.

${ }^{27}$ Maspero fut directeur du Service de 1881 à 1886, puis fut remplacé par Grébaut (1886), De Morgan (1892), et Loret (1897), avant de revenir à la tête du Service pour un second mandat, de 1899 à 1914. Sur la transition entre les directions de Loret et de Maspero, voir Goyon, Kyphi 5 (2006) et David (éd.), Gaston Maspero, p. 190-92.

${ }^{28}$ Sur ce point, voir Gran-Aymerich, Les chercheurs de passé 1798-1945, p. 371, ainsi que Petricioli dans La Rosa (éd.), L'archeologia italiana nel Mediterraneo, p. 15 : «E quindi lecito ipotizzare che Maspero, e la autorità francesi, vedessero nella concessione all'Italia non solo un modo per sanzionare i nuovi rapporti tra i due paesi ma anche la via per riequilibrare lo strapotere britannico in Egitto ».

${ }^{29}$ Reid, Whose Pharaohs ?, p. 113-16.

${ }^{30}$ Reid, Whose Pharaohs ?, p. 197-98. Les Allemands sont entre - temps occupés en Turquie sur le plan archéologique, conséquence de leur présence militaire et leur construction d'infrastructures dans la région du croissant fertile. Les fouilles de Pergame en sont 
un bon exemple.

${ }^{31}$ Ainsi, il y avait bien des égyptologues étrangers, autres que Français, au sein du Service des Antiquités avant la direction Maspero, tels les Italiens Luigi Vassalli (présent entre 1859 et 1883) et Alexandre Barsanti (1881-1917), ou l'Allemand Emile Brugsch (18721913). Mais ils travaillaient au nom du Service, et non comme équipe de fouille au service d'une institution représentant leur pays, comme ce fut le cas par la suite.

${ }^{32}$ Reid, Whose Pharaohs ?, p. 176.

${ }^{33}$ Maspero, Rapports 1899-1910.

${ }^{34}$ Sur ce point, voir Khater, Le régime juridique des fouilles, p. 282, annexe VIII. « Décret du 17 décembre 1891 arrêtant les conditions auxquelles le autorisations de fouilles peuvent être délivrées. (...) Art. 2. Tous les objets trouvés dans les fouilles appartiennent de droit à l'Etat et doivent être déposés au musée de Guizeh ».

${ }^{35}$ Maspero, Rapports 1899-1910, p. XXX.

36 « Il faut me livrer à ces travaux en un moment critique (...) où tous les temples arrivent à l'échéance : ils menacent en ce moment de s'écrouler, alors que le temps et l'argent font défaut ; il est tel temple que les touristes traversent pour se rendre aux tombeaux des rois dont les pierres sont prêtes à tomber sur les passants, qui ne savent à quels périls ils s'exposent. Dans ces conditions je me suis réservé les soins de la restauration des grands monuments, abandonnant les autres recherches aux particuliers et aux associations fondées pour explorer les sites antiques ». Maspero, L’Ami des monuments français 21 (1908), p. 80.

${ }^{37}$ Tableau établi à partir des données des Rapports, de 1903 à 1912, rédigés par Gaston Maspero. Les sites pouvant désigner, selon leur échelle, des localisations plus ou moins étendues, voici la liste des dénominations de lieux comptant pour un site de fouille dans le tableau ci-dessus, établie à partir des dénominations données par Maspero lui-même : Abousir, Abou Mena, Abydos, Amarna, Antinoé, Aphroditopolis Magna, Assiout, Assouan, Athribis, Badari, Baouit, Beni Hassan, Biban el-Moulouk, Coptos, Deir el-Bachit, Deir el-Bahari, Deir el-Medina, Dendéra, Dra Abou el-Naga, Edfou, El Bahnasa, El-Assasif, Eléphantine, El-Hiba, El-Hammayia, Esna, Gebel Silsila, Gebelein, Giza, Gourna, Gournet Mourraï, Hawara, Héliopolis, Hérakleopolis Magna, Hermopolis Magna (Ashmounein), Hiérakonpolis (nécropole), Karnak, Kharga, Kom Ombo, Le Fayoum, Licht, Médinet Habou, Meïdoum, Memphis, Mit-Rahina, Montagne thébaine, Naga ed-Deir, Nubie, Ouadi Hamamat, Péluse, Philae, Qaw el-Kebir, Samaria, Sinai, Tarkhan, Tehneh, Tell el-Maskhouta, Tell el-Yahoudi, Thèbes, Thèbes - Vallée de l'Ouest, Thinis, Touna el-Gebel, Vallée des Reines, Vallée des Rois, Xois, Zaouiet el-Aryan.

${ }^{38}$ Gady, « Le pharaon, l'égyptologue et le diplomate », p. 635-36.

39 « [...] face à la concurrence étrangère dans ce domaine, la France ne pouvait plus prétendre tenir la première place. De 1899 à 1913, les Français réalisèrent un quart des travaux mentionnés par Maspero, alors que les Britanniques en avaient réalisé plus d'un tiers », Gady, « Le pharaon, l'égyptologue et le diplomate », p. 634.

${ }^{40}$ Dans une lettre datée du 3 novembre 1904, Maspero relate une conversation avec Lord Cromer à propos du choix de son successeur à la tête du Service, où il rappelle à celui-ci que le candidat doit être français, comme convenu par l'Entente Cordiale. Cromer accepte après s'être assuré que les deux autres employés du Service, les inspecteurs en chef, resteront des Anglais (Quibell et Carter). On constate une sorte d'équilibre précaire que chaque partie tente de maintenir, entre mainmise française et britannique, puisque la nationalité du candidat se révèle être un aspect presque aussi important que ses compétences dans le choix du directeur du Service. Voir David (éd.), Gaston Maspero.

${ }^{41}$ Voir Stevenson, in Journal of the History of Collection 26/1 (2013), p. 89-102. Stevenson explore " the developement of a 'scientific' archaeology in Egypt » (p. 6) en corrélation avec le développement de la notion d'« excavated artefact », soit d'un résultat de fouilles, une pièce scientifique qui remplace peu à peu, au tournant du siècle, l'idée d'une antiquité ou d'un simple objet esthétique.

${ }^{42}$ L'IFAO se nomme alors, et jusqu'en 1898, Mission Archéologique du Caire. Maspero ne le dirige que pendant l'année 1880, puis quitte officiellement le poste pour prendre la suite de Mariette à la tête du Service des Antiquités en 1881, néanmoins, les liens sont forts entre les deux organismes, bien que l'un soit au service de l'Egypte et l'autre de la France : dans les faits, les directeurs des deux institutions proviennent de la même école, trois d'entre eux ont d'ailleurs successivement dirigé l'IFAO puis le Service, Maspero, Grébaut, puis Lacau.

${ }^{43}$ Curto, dans Moiso (éd.), Ernesto Schiaparelli e la tomba di Kha, p. 40.

${ }^{44}$ Moiso, La storia del Museo Egizio, p. 58-60.

${ }^{45}$ Moiso, dans Del Vesco et Moiso (éd.), Missione Egitto, p. 150.

${ }^{46}$ Première fois au nom de l'Italie en tant qu'Etat-Nation. Voir n. 6.

${ }^{47}$ Bien que nous n'ayons pas, à notre connaissance, de documents témoignant des pensées de Schiaparelli sur cette question, le problème de l'achat de faux était très présent à l'esprit des égyptologues et conservateurs venant acquérir des objets en Egypte. En témoigne, par exemple, cette remarque de Maspero sur son collègue allemand Adolf Erman, en 1886 : «L'approche d'Erman a jeté l'émoi dans le pays : partout on lui prépare les plus jolis objets faux qu'on peut trouver. Sa réputation comme acheteur est faite du Caire : il passe pour payer très cher et pour ne pas savoir reconnaître le faux du vrai. Le fait est qu'il a enrichi le musée de Berlin de diverses antiquités fort modernes (...) », David (éd.), Gaston Maspero, p. 140. Un autre exemple montrant l'importance de ce problème étroitement lié à l'achat d'objets sur le marché de l'art égyptien nous est donné dans le contexte de l'ouverture de la Salle des Ventes du Musée de Giza en 1892. Outre les avantages certains pour le Service 
des Antiquités, De Morgan dans ses mémoires évoque également l'intérêt d'une telle démarche pour les acheteurs eux-mêmes, étant ainsi « certains de ne point être trompés », cité dans Piacentini, Forming Material Egypt, p. 112.

${ }^{48}$ Fond principal du Museo Egizio avant Schiaparelli, acquis en 1824 .

${ }_{49}^{49}$ Maspero fut le premier à les étudier, en 1880 , et à les identifier comme provenant de Deir el-Medina. Maspero, « Rapport sur une mission en Italie », dans RecTrav p. 159-99.

${ }^{50}$ Schiaparelli, Relazione sui lavori, II, préface.

${ }^{51}$ Numéro d'inventaire Cat. 1618.

${ }^{52}$ Lettre de Schiaparelli au Ministre de l'Instruction Publique, 29 avril 1902. Turin, Archivio di Stato, MAE, M1 n5, Prot. 3048. Voir Moiso, dans Del Vesco et Moiso (éd.), Missione Egitto, p. 150.

${ }^{53}$ Ibid., « tutte indistintamente le principali Nazioni Europee », « contribuire insieme alle più dotte Nazioni allo studio di una civiltà ».

${ }^{54}$ Ibid., « L'Italia [...] che ha gloriose tradizioni scientifiche $»$.

${ }^{55}$ Ibid., « (...) una civiltà, la cui importanza nella storia si rivela ogni giorno maggiore ».

${ }^{56}$ Ibid., Schiaparelli écrit au Ministre : « Ora mi fa pregio rimettere copia a V.E di una lettera [...] dalla quale risulta che, memore di un mio antico desiderio, e con singolare deferenza per questo Museo, il Maspero, Direttore Generale delle Antichità in Egitto, avrebbe riservato al Museo di Torino la ricerca e lo scavo della necropoli di Eliopoli, e di tombe reali nella Valle delle Regine. ». Il reprend cette idée d'invitation plus loin dans la lettre « Lo scavo che, forse per l'ultima volta, ci viene proposto è di tale importanza (...) », ou encore « L'Italia [...] non deve ne può ricusare l'invito che le viene forse fatto per l'ultima volta ». Dans les faits, il avait auparavant envoyé une demande d'autorisation de fouilles à Maspero, et le courrier mentionné comme une invitation de sa part est en réalité une réponse positive à cette demande : «J'ai l'honneur de vous informer que le Comité d'Egyptologie a accordé l'autorisation que vous avez demandé » (Lettre officielle de Maspero à Schiaparelli, 20 avril 1902, Torino, Archivio di Stato, MAE, M1n5, Prot. 389 ; Del Vesco, dans Del Vesco et Moiso [éd.], Missione Egitto, p. 137, no. 118).

${ }^{57}$ Ibid., « Per quanto concerne la parte finanziaria, non vi sarebbero difficoltà tali, che non possano essere superate $»$.

${ }^{58}$ Giza, la Vallée des Reines, Deir el-Medina, Héliopolis (Matariya), Hermopolis Magna (Ashmounein), el-Hammamiya, Antaeopolis (Qaw el Kebir), Assiout, Gebelein, El Bahnasa (Ossrinco), et Assouan. Voir Moiso (éd.), Ernesto Schiaparelli e la tomba di Kha, p. 268.

${ }^{59}$ Moiso, La storia del Museo Egizio, p. 74.

${ }^{60}$ Moiso, La storia del Museo Egizio, p. 60.

${ }^{61}$ Maspero, « Année 1903 », dans Rapports 1899-1910, p. 101. Del Vesco, dans Del Vesco et Moiso (éd.), Missione Egitto, p. 225.

${ }^{62}$ Maspero, « Année 1903 », dans Rapports 1899-1910, p.101.
${ }^{63}$ Donadoni et Donadoni Roveri, L'Egitto dal mito all'egittologia, p. 261. Cette « difficulté » est mentionnée mais non expliquée, il est seulement dit que l'incident fut résolu avec l'aide de Maspero et la diplomatie de Breccia en divisant le terrain en parts égales.

${ }^{64}$ « M. Breccia a travaillé quelques semaines à Echmounéîn, et il y a recueilli des fragments de papyrus assez nombreux : toutefois des difficultés, survenues entre lui et M. Rubensohn au sujet de leurs concessions respectives, ne lui ont pas permis de donner à ses opérations toute l'étendue qu'elles comportaient. » Maspero, « Année 1903 », dans Rapports 1899-1910, p. 102.

${ }^{65}$ Voir Maspero, ASAE 1 (1900), p. 1-2 : «J'estime qu'on m'approuvera d'écarter des Annales tous les articles de pure discussion ou de critique scientifique: les journaux publiés en Europe [...] ont plus qualité qu'elles pour les recevoir. Je crois qu'en revanche elles rendront des services réels à notre science, si l'on y insère chaque année les rapports des Inspecteurs généraux et locaux, sur les fouilles qu'ils auront dirigées ou sur les sites qu'ils auront parcourus au cours de leurs inspections, ainsi que la reproduction presque sans commentaire des inscriptions ou des objets qui seront sortis des travaux entrepris par la Direction ».

${ }^{66}$ Loret commence le projet alors qu'il est encore Directeur Général des Antiquités, et c'est Maspero, à son retour en 1899, qui le reprend et publie le premier numéro en 1900.Voir Maspero, ASAE 1 (1900).

67 « For the newly discovered tombs of Nefertari-Meri-Mut and Seth-hi-khopesh-ef, arches have been built over the entrances to preserve them against rain water or falling stones; iron plated doors are being made, and I hope to have them fixed before the end of the year. I have made the experiment here of a new design of iron door hitherto used for tombs (...) », Carter et Legrain, ASAE 6 (1905), p. 120.

${ }^{68}$ Maspero, «Année 1903 », dans Rapports 1899-1910, p. 101.

${ }^{69}$ Voir Maspero, ASAE 1 (1900).

${ }^{70}$ Maspero, «Année 1904 », dans Rapports 1899-1910, p. 128.

${ }^{71}$ Maspero, « Année 1905 », dans Rapports 1899-1910, p. 176.

${ }^{72}$ Maspero, «Année 1905 », dans Rapports 1899-1910, p. 176.

${ }^{73}$ Maspero, « Année 1906 », dans Rapports 1899-1910, p. 209. La tombe de Kha date de la XVIII ème dynastie, mais a d'abord été datée de la XX⿳亠丷⿵冂丶 ${ }^{2}$ par Ernesto Schiaparelli, avis suivi ici par Maspero.

${ }^{74}$ D’après Moiso, La storia del Museo Egizio, p.64.

${ }^{75}$ Source : analyses du contenu des ASAE de 1903 à 1912.

${ }^{76} 15.79 \%$ de contributeurs Allemands et $5.26 \%$ de contributeurs américains.

${ }^{77}$ Schiaparelli, ASAE 21 (1921), p. 126-28. Le nombre de page indique une contribution succincte, outre le fait qu'elle soit l'unique rédigée par Schiaparelli.

${ }^{78}$ Maspero, « Année 1907 », dans Rapports 1899-1910, p. 235.

${ }^{79}$ Maspero, «Année 1910 », dans Rapports 1899-1910, 
p. 325.

${ }^{80}$ Pour le Rapport du Service des Antiquités, nous avons repris les données du document 1, et pour les Annales, nous avons analysé les 290 articles publiés dans les numéros 4 à 12 des ASAE, dont 85 traitaient ou faisaient mention de fouilles effectuées en Egypte sur la période étudiée.

${ }^{81}$ Voir p. 8 de cet article.

${ }^{82}$ Il est vrai que le Service emploie également quelques égyptologues de nationalités allemande et britannique, mais ils sont bien moins nombreux.

${ }^{83}$ Reid, Whose Pharaohs ?, p. 197.

${ }^{84}$ Borchardt se voit interdit de fouiller par Lacau jusqu'à ce qu'il soit remplacé en 1929 par Hermann Junker.

${ }^{85}$ Pour le compte du Musée de Budapest, menées par le polonais Smolenski.

${ }^{86}$ Schiaparelli, Relazione sui lavori, I.

${ }^{87}$ Schiaparelli, Relazione sui lavori, II.

88 « Il presente volume contiene solamente la relazione degli scavi eseguiti nella Valle delle Regine negli anni 1903-1905, ed appare con tanto ritardo per contrattempi molteplici, più particolarmente dovuti alla esecuzione del lavoro eliotipico; per cui, sebbene la stampa ne sia stata iniziata fin dall'anno 1914, solo nello scorcio del corrente anno 1923 ne è stato possibile il compimento. L'essere stata iniziata la presente pubblicazione prima della guerra, in un periodo nel quale le pubblicazioni della natura della presente si solevano eseguire con abbondanza di illustrazioni e in grande formato, giustificherà il lusso della medesima, che in questo periodo del dopo guerra non sarebbe altrimenti giustificato ", Schiaparelli, Relazione sui lavori, I, préface.

${ }^{89}$ Lettre d'avertissement concernant les publications : communication du Service (P. Lacau) aux archéologues du 3 juin 1922, Torino, Archivio di Stato, MAE, 20 vers., M1 n21. Del Vesco, dans Del Vesco et Moiso (éd.), Missione Egitto, p. 139, no. 122.

${ }^{90}$ Voir Khater, Le régime juridique des fouilles, p.174.

${ }^{91}$ Calderini, Aegyptus, 8/3-4 (1928), p. 337-38. Ce dernier mentionne néanmoins deux références abrégées de possibles publications par Schiaparelli que nous n'avons pu identifier ou retrouver : « Schiaparelli, Rass. Naz. 69 (1882), p. 3 », ainsi que «Boll. P. Istr. 15 settembre 1904.».

${ }^{92}$ Gebelein, même site qui avait fait, lors d'une précédente mission, l'objet du seul rapport par Schiaparelli dans les ASAE. Voir n. 75.

${ }^{93}$ Excepté une paire de lampes de sol, selon Gady, « Le pharaon, l'égyptologue et le diplomate », p. 632.

${ }^{94}$ Gran-Aymerich, Les chercheurs de passé 1798-1945, p. 332. Lauteur poursuit : " Avant la Grande Guerre, l'Égypte donne l'exemple d'une exploration archéologique concertée et d'une coopération que les protectorats anglais et français, établis en Proche Orient après 1918, favoriseront ».

\section{Bibliographie}

Bednarski, Andrew, « The nature and history of Egyptology », dans Ian Shaw et Elizabeth Bloxam (éd.), The Oxford Handbook of Egyptology, Juin 2015, DOI: https://doi.org/10.1093/oxford- hb/9780199271870.013.2 (en ligne).

Bonacasa, Nicola, « L’Archeologia italiana in Egitto », dans: Vincenzo La Rosa, (éd.), L'archeologia italiana nel Mediterraneo fino alla seconda guerra mondiale, Catania, 1986, pp. 41-52.

Calderini, Aristide, « Ernesto Schiaparelli », Aegyptus, 8/3-4 (1928), p. 337-38.

Carter, Howard et Georges Legrain, « Report of Work Done in Upper Egypt (1903-1904)», ASAE 6 (1905), p. 112-29.

Curto, Silvio, « Schiaparelli e il suo tempo », dans Beppe Moiso (éd.), Ernesto Schiaparelli e la tomba di Kha, p. 35-45.

David, Elisabeth, Mariette Pacha 1821-1881, Paris, 1994.

David, Elisabeth, Gaston Maspero 1846-1916. Le gentleman égyptologue, Paris, 1999.

David, Elisabeth (éd.), Gaston Maspero, Lettres d’Égypte. Correspondance avec Louise Maspero 1883-1914, Paris, 2003.

David, Elisabeth, « Un brouillon de Mariette. Un projet pour un Service des Antiquités de l'Égypte international », Revue d 'égyptologie 61 (2010), pp. 209-224.

De Gayffier-Bonneville, Anne-Claire, Histoire de l'Egypte moderne. L'éveil d'une nation, XIX-XX'̀me siècles, Paris, 2016.

Del Vesco, Paolo, « All'ombra delle piramidi : gli scavi italiani a Giza », dans Paolo Del Vesco et Beppe Moiso (éd.), Missione Egitto 1903-1920 : l'avventura archeologica M.A.I. raccontata, pp. 223-230.

Del Vesco, Paolo, « Comunicazione da parte del Service des Antiquités con le regole per gli archeologi », dans Paolo Del Vesco et Beppe Moiso (éd.), Missione Egitto 1903-1920 : l'avventura archeologica M.A.I. raccontata, p. 139 , no. 122.

Del Vesco, Paolo, « Lettera ufficiale di Gaston Maspero a Ernesto Schiaparelli riguardante il permesso di scavo », dans Paolo Del Vesco et Beppe Moiso (éd.), Missione Egitto 1903-1920 : l'avventura archeologica M.A.I. raccontata, p. 137, no. 118.

Del Vesco, Paolo et Beppe Moiso (éd.), Missione Egitto 1903-1920 : l'avventura archeologica M.A.I. raccontata, Modena, 2017.

Donadoni, Sergio, Silvio Curto, et Anne Maria Donadoni Roveri, L'Egitto dal mito all'egittologia, Milano, 1990.

Gady, Eric, « Le pharaon, l'égyptologue et le diplomate. Les égyptologues français en Egypte, du voyage de Champollion à la crise de Suez », (thèse de doctorat non publiée, sous la direction de Jacques Frémeaux, Université Paris IV-Sorbonne), Paris, 2005.

Gady, Eric, « L’archéologie de l’Égypte antique pendant la période coloniale de l'occupation britannique à la découverte du tombeau de Toutankhamon ", Les nouvelles de l'archéologie 126 (2011), DOI : https://doi. org/10.4000/nda.1195.

Goyon, Jean-Claude, « Victor Loret administrateur, 1897-1899 et l’Égypte sous tutelle Britannique », Kyphi 5 (2006), p. 83-86.

Gran-Aymerich, Eve, Les chercheurs de passé 1798-1945, Paris, 2007.

Khater, Antoine, Le régime juridique des fouilles et des antiquités en Égypte, Le Caire, 1960. 
Maspero, Gaston, « Rapport sur une mission en Italie », in RecTrav, Le Caire, 1880, p. 159-99.

Maspero, Gaston, « Avertissement », ASAE 1 (1900), p. 1-2.

Maspero, Gaston, « Le Barrage du Nil et la destruction et la conservation des monuments de l'Égypte », L'Ami des monuments français 21 (1908), p. 77-80.

Maspero, Gaston, Rapports sur la marche du Service des Antiquités 1899-1910, Le Caire, 1912.

Maspero, Gaston, Rapports sur la marche du Service des Antiquités 1911, Le Caire, 1912.

Maspero, Gaston, Rapports sur la marche du Service des Antiquités 1912, Le Caire, 1913.

Miège, Jean-Louis, L'Impérialisme colonial italien de 1870 à nos jours, Paris, 1968.

Moiso, Beppe (éd.), Ernesto Schiaparelli e la tomba di Kha, Torino, 2008.

Moiso, Beppe, « La fondazione della missione e la richiesta di fondi », dans Paolo Del Vesco et Beppe Moiso (éd.), Missione Egitto1903-1920 : l'avventura archeologica M.A.I. raccontata, p. 149-52.

Moiso, Beppe, La storia del Museo Egizio, Modena, 2016.

Petricioli, Marta, « Le missioni archeologiche italiane nei paesi del Mediterraneo : uno strumento di politica internazionale », dans Vincenzo La Rosa (éd.), L'archeologia italiana nel Mediterraneo fino alla seconda guerra mondiale, Catania, 1986, p. 9-32.

Piacentini, Patrizia (éd.), Victor Loret in Egypt (18811899), Le Caire, 2008.

Piacentini, Patrizia, « The Antiquities Path », dans Patrizia Piacentini (éd.), Forming Material Egypt, Milan, 2013, p. 105-30.

Reid, Donald, Cairo University and the Making of Modern Egypt, Cambridge 1990.

Reid, Donald, « Nationalizing the Pharaonic Past:
Egyptology Imperialism and Egyptian Nationalism, 1922-1952 », dans James Jankowski et Israel Gershani (éd.), Rethinking Nationalism in the Arab Middle East, New York, 1997, p. 127-49.

Reid, Donald, « The Urabi revolution and the British conquest, 1879-1882 », dans Martin Daly (éd.), The Cambridge History of Egypt, II , Cambridge, 1998, p. 217-38.

Reid, Donald, Whose Pharaohs ?, Cairo, 2002.

Reid, Donald, Contesting Antiquity in Egypt, Cairo, 2015. Schiaparelli, Ernesto, « La Missione italiana a Gebelein », ASAE 21 (1921), p. 126-28.

Schiaparelli, Ernesto, Relazione sui lavori della Missione archeologica italiana in Egitto (anni 1903-1920), I: Valle delle Regine, Torino, 1923.

Schiaparelli, Ernesto, Relazione sui lavori della Missione archeologica italiana in Egitto (anni 1903-1920), II: la tomba intatta dell'architetto Kha, Torino, 1927.

Service des Antiquités Egyptiennes (éd.), ASAE 4 à 12 (1903-1912).

Stevenson, Alice, « Between the Field and the Museum », dans Patrizia Piacentini (éd.), Forming Material Egypt, Milan, 2013, p. 143-54.

Stevenson, Alice, « Artefacts of Excavation: The British Collection and Distribution of Egyptian Finds to Museums, 1880-1915 », Journal of the History of Collection 26/1 (2013), p. 89-102, DOI: https://doi. org/10.1093/jhc/fht017.

Stevenson, Alice, The Petrie Museum of Egyptian Archaeology, Characters and Collections, London, 2015.

Thompson, Jason, Wonderful Things : A History of Egyptology, II, The Golden Age 1881-1914, Cairo,2015.

Trümpler, Charlotte (éd.), Das Große Spiel : Archäologie und Politik zur Zeit des Kolonialismus (1860-1940), Cologne, 2008. 